\title{
Parameterizing the instrumental spectral response function and its changes by a super-Gaussian and its derivatives
}

\author{
Steffen Beirle ${ }^{1}$, Johannes Lampel ${ }^{1, a}$, Christophe Lerot ${ }^{2}$, Holger Sihler ${ }^{1}$, and Thomas Wagner ${ }^{1}$ \\ ${ }^{1}$ Max-Planck-Institut für Chemie (MPI-C), Satellite Remote Sensing Group, Mainz, Germany \\ ${ }^{2}$ Royal Belgian Institute for Space Aeronomy (BIRA-IASB), Brussels, Belgium \\ ${ }^{a}$ now at: Institut für Umweltphysik (IUP), Universität Heidelberg, Heidelberg, Germany
}

Correspondence to: Steffen Beirle (steffen.beirle@mpic.de)

Received: 20 September 2016 - Discussion started: 18 October 2016

Revised: 17 January 2017 - Accepted: 31 January 2017 - Published: 23 February 2017

\begin{abstract}
The instrumental spectral response function (ISRF) is a key quantity in DOAS analysis, as it is needed for wavelength calibration and for the convolution of trace gas cross sections to instrumental resolution. While it can generally be measured using monochromatic stimuli, it is often parameterized in order to merge different calibration measurements and to plainly account for its wavelength dependency. For some instruments, the ISRF can be described appropriately by a Gaussian function, while for others, dedicated, complex parameterizations with several parameters have been developed.

Here we propose to parameterize the ISRF as a "superGaussian", which can reproduce a variety of shapes, from point-hat to boxcar shape, by just adding one parameter to the "classical" Gaussian. The super-Gaussian turned out to describe the ISRF of various DOAS instruments well, including the satellite instruments GOME-2, OMI, and TROPOMI.

In addition, the super-Gaussian allows for a straightforward parameterization of the effect of ISRF changes, which can occur on long-term scales as well as, for example, during one satellite orbit and impair the spectral analysis if ignored. In order to account for such changes, spectral structures are derived from the derivatives of the super-Gaussian, which are afterwards just scaled during spectral calibration or DOAS analysis. This approach significantly improves the fit quality compared to setups with fixed ISRF, without drawbacks on computation time due to the applied linearization. In addition, the wavelength dependency of the ISRF can be accounted for by accordingly derived spectral structures in an easy, fast, and robust way.
\end{abstract}

\section{Introduction}

The instrumental spectral response function (ISRF), also denoted as "instrument transfer function" or just "slit function", describes the spectral response to a monochromatic stimulus and is thus a key quantity in spectroscopy. Within differential optical absorption spectroscopy (DOAS) (Platt and Stutz, 2008), good knowledge of the ISRF is needed in order to perform an accurate wavelength calibration of the instrument and to convolve the relevant absorption cross sections on the instrument's spectral resolution.

The ISRF is determined by the optical properties of the instrument (entry and exit slits, gratings, detector properties etc.) but is typically too complex to be accurately reproduced by a physical model. It can, however, generally be measured accurately in the laboratory using quasimonochromatic stimuli ${ }^{1}$ like spectral light sources (SLSs) combining different atomic emission lines, light that has passed a monochromator, or a (tunable) laser.

The measured ISRF might be directly applied to the convolution of high-resolution trace gas cross sections. Often, however, the ISRF is parameterized by an appropriate function in order to (a) merge different calibration measurements, (b) describe the wavelength dependency of the ISRF by wavelength-dependent parameters, and (c) determine the ISRF directly from measurements of direct or scattered sunlight, making use of the highly structured Fraunhofer lines.

\footnotetext{
${ }^{1}$ For the instruments considered in this study with moderate spectral resolutions (some $0.1 \mathrm{~nm}$ ), the line width of the stimuli is usually negligible.
} 
One of the simplest possible parameterizations of the ISRF is a Gaussian, which often describes the measured line shapes fairly well by only one free parameter, $\sigma$, plus an asymmetry parameter if needed. This parameterization works well, e.g., for the Global Ozone Monitoring Instrument 2 (GOME2) (Munro et al., 2016). For the Ozone Monitoring Instrument (OMI) (Levelt et al., 2006), however, a Gaussian parameterization of the ISRF is not appropriate. Instead, the OMI ISRF was parameterized by a sum of a Gaussian and a "broadened Gaussian" with an exponent of 4 instead of 2 (Dirksen et al., 2006). Both summands can have different amplitude, width, and shift relative to each other, such that the final parameterization can describe different shapes, including asymmetric patterns. A similar parameterization of the ISRF was proposed by Liu et al. (2015) for an aircraft instrument used during the DISCOVER-AQ campaign. For the upcoming TROPOMI instrument (Veefkind et al., 2012) on the Sentinel 5 Precursor (S-5P) mission, the ISRF was parameterized by an "advanced sigmoid" with nine parameters for the ultraviolet (UV) and near-infrared (NIR) and a "generalized exponential" (composed of broadened Gaussians with different exponents) with eight parameters for the UV-visible (UVIS) band (J. Smeets, A. Ludewig, Q. Kleipool, Calibration analysis report for TROPOMI UVN ISRF, personal communication, 2016).

Such tailored parameterizations have been demonstrated to reproduce the measured line shapes well. However, parameterizations using many parameters generally introduce ambiguities, leading to parameters being (anti-)correlated (in the sense that changes of different parameters can cause very similar responses of the ISRF). Thus, while these complex parameterizations can be fitted well to a measured monochromatic stimulus, a fit within wavelength calibration based on measured Fraunhofer lines is generally challenging, as ambiguities result in slow and often unstable fits.

In this study, we propose to use a modification of the Gaussian, the so-called "super-Gaussian" (SG), for the parameterization of the ISRF. The SG has long been in use in laser physics to describe flat-topped beam distributions (e.g., Fleck et al., 1977; Decker, 1995). Nadarajah (2005) denotes it as generalized normal distribution and provides an overview of its mathematical characteristics. Recently, it has also been used to describe the spatial field of view of OMI (de Graaf et al., 2016; Sihler et al., 2016).

The SG can reflect a wide variety of shapes by adding just one parameter compared to the classical Gaussian, and thus it is still a rather simple, but powerful, extension. Within the DOAS community, the parameterization of the ISRF by an SG is already implemented in Pandora (Cede, 2013) and Blick software (Cede, 2015) and is planned to be implemented in QDOAS in a next release (C. Fayt, personal communication, 2016).

In the second part of this study, we focus on ISRF changes. While the ISRF can usually be measured with high accuracy in the lab, the ISRF might change over time, in particular if the instrument is moved or if conditions like temperature change.

In particular, for GOME-2 the ISRF has changed significantly over time, as shown in Munro et al. (2016), which turns out to be related to temperature changes. Such changes of the ISRF cause a highly structured spectral response, which impair the spectral analysis of trace gases if not accounted for. Thus, significant improvement of derived HCHO columns and ozone profiles are reported by, e.g., De Smedt et al. (2012) and Miles et al. (2015), respectively, after fitting the GOME-2 ISRF based on daily irradiance measurements rather than taking the key data based on preflight measurements.

Recently, also the impact of changing ISRF over one satellite orbit has been addressed. Azam and Richter (2015) reported a systematic increase of the GOME-2 ISRF width along one orbit by determining the ISRF, parameterized as a Gaussian, within a nonlinear fit of a high-resolution solar atlas, convolved with the ISRF, to the measured radiance for each satellite pixel. Instead of such a time-consuming nonlinear fit, which is not feasible for operational analysis of longer time series, the spectral effects of an ISRF change can also be accounted for by adding a "pseudo-absorber" (PA) to the spectral analysis, which is derived from the difference of two spectra convolved with slightly modified (squeezed) ISRF (denoted as "resolution correction" by Azam and Richter, 2015; see Sect. 4.4 therein). Similar findings have been reported by van Roozendael et al. (2014), who used PAs accounting for ISRF changes in order to improve fit quality and to monitor instrument stability (M. van Roozendael, personal communication, 2016).

Here we formally extend this approach and deduce the spectral effects caused by ISRF changes by a Taylor expansion. This allows for a linearization within the spectral analysis by inclusion of spectral structures which are just scaled within the fit procedure. Linearization leads to more stable fits, as it excludes local side minima of the residual function, and allows for a much faster DOAS analysis, as soon as all relevant effects such as spectral shifts are linearized (Beirle et al., 2013).

While the presented Taylor expansion is given in general form, it is applied for the SG parameterization, which is particularly suited due to the limited number of parameters which are uncorrelated and have a descriptive meaning (width and shape).

In this study we

- introduce the SG and its properties in Sect. 2.1 and derive a general formalism for the effects of ISRF changes in Sect. 2.2;

- demonstrate how far the SG is capable of reproducing ISRFs (Sect. 4) for different instruments introduced in Sect. 3; 
- give examples of applications of the linearized treatment of ISRF changes and the benefit for wavelength calibration and trace gas retrievals in Sect. 5 .

\section{Methods}

\subsection{The super-Gaussian}

The normalized Gaussian function, $G$, is given as

$G(x)=\frac{1}{\sqrt{2 \pi} \sigma} e^{-\frac{1}{2}\left(\frac{x}{\sigma}\right)^{2}}$.

The super-Gaussian, $S$, can be expressed as

$S(x)=A(w, k) \times e^{-\left|\frac{x}{w}\right|^{k}}$,

with two independent parameters, $w$ and $k$, determining the width and shape of $S$, respectively. This formulation is equivalent to the generalized normal distribution discussed in Nadarajah (2005).

For application of $S$ as ISRF, it has to be normalized to an integral of 1 via $A(w, k)$. In case of infinite bounds, $A(w, k)$ can be expressed as (Nadarajah, 2005)

$A(w, k)=\frac{k}{2 w \Gamma\left(\frac{1}{k}\right)}$,

where $\Gamma$ is the gamma function; i.e., $A(w, k)$ is proportional to the inverse width, like for $G$, and depends slightly on $k$ with a maximum for $k=2$. In practice, however, the ISRF has to be defined and applied on a finite interval. Thus, within the application of $S$ as ISRF parameterization, it has to be normalized on a finite interval as well. The finite integrals needed for normalization, as well as the partial derivatives of $S$ required in the next section, are thus determined numerically.

Figure 1 displays $S$ for different values of the "shape parameter" $k$. For $k=2, S$ equals $G$ with

$w_{k=2}=\sqrt{2} \sigma$.

For $k>2, S$ becomes "flat-topped", converging to a boxcar shape of width $2 w$ for $k \rightarrow \infty$. For $k<2, S$ becomes more peaked at the top, with long tails on both sides.

The SG as defined in Eq. (2) is symmetric by definition but can easily be extended to an asymmetric SG (ASG) by introducing asymmetry parameters for $w$ and/or $k$, as described in Appendix A. In this study, we focus on symmetric SG $S$ or the asymmetric extension $S_{a_{w}}$ with the additional parameter $a_{w}$ determining the asymmetry of its width (see Appendix A for details).

The full width at half maximum (FWHM), which is often used as measure for the width of a distribution, is

$\mathrm{FWHM}=2 \sqrt[k]{\ln 2} w$,

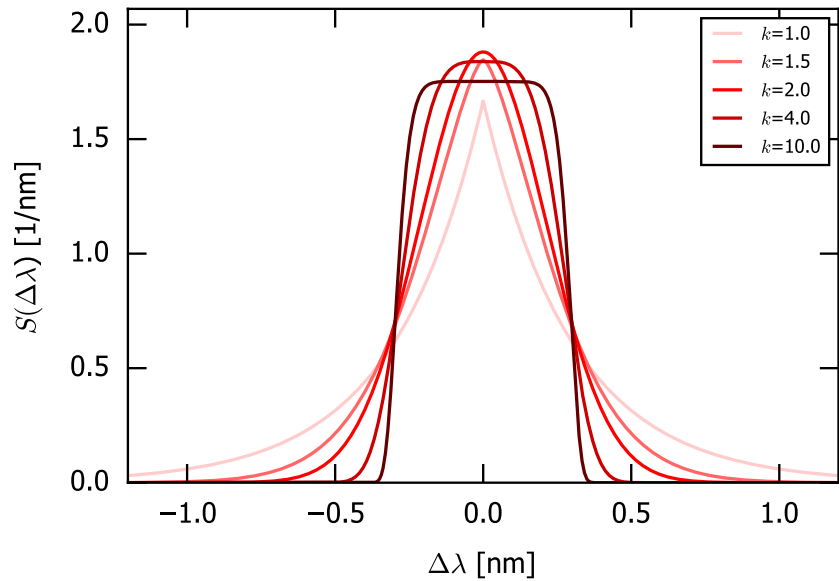

Figure 1. Illustration of the "super-Gaussian" as defined in Eq. (2) for $w=0.3 \mathrm{~nm}$ and different values of the shape parameter $k$.

i.e., it depends on $k$. For the SG, it is thus useful to consider the "full width at $1 / \mathrm{e}^{\text {th }}$ maximum" (FWEM) instead, as this directly corresponds to $w$ doubled:

FWEM $=2 w$,

which holds independent of $k$, even in the asymmetric case (see Appendix A).

\subsection{Parameterizing ISRF changes}

Changes of the ISRF cause a spectral response to the measured spectra. In particular for direct or scattered sunlight, this response is highly structured due to the Fraunhofer lines. Such spectral structures impair spectral analyses like DOAS, resulting in larger fit residuals, larger statistical errors of fitted column densities, and possibly also systematic biases if not appropriately accounted for.

In this section we show that the spectral structures caused by a change of the ISRF can be linearized with respect to the parameter change and thus can be accounted for by adding correction spectra to the spectral analysis. This generally makes the fit more stable, as local side minima are excluded, and significantly faster.

\subsubsection{Change of ISRF}

Be $P(p)$ a general parameterization of the ISRF with parameter $p$. In order to parameterize the effect of changes of $p$, we determine the Taylor expansion of $P$ around the baseline $P^{*}=P\left(p^{*}\right)$ for a change of $\Delta p=p-p^{*}$ :

$$
\begin{aligned}
P(p, \lambda) & =P\left(p^{*}, \lambda\right)+\left.\Delta p \frac{\partial P(p, \lambda)}{\partial p}\right|_{p^{*}}+\mathcal{O}(2) \\
& =P^{*}+\Delta p \times \partial_{p} P+\mathcal{O}(2),
\end{aligned}
$$

with

$\partial_{p} P:=\left.\frac{\partial P(p, \lambda)}{\partial p}\right|_{p^{*}}$ 


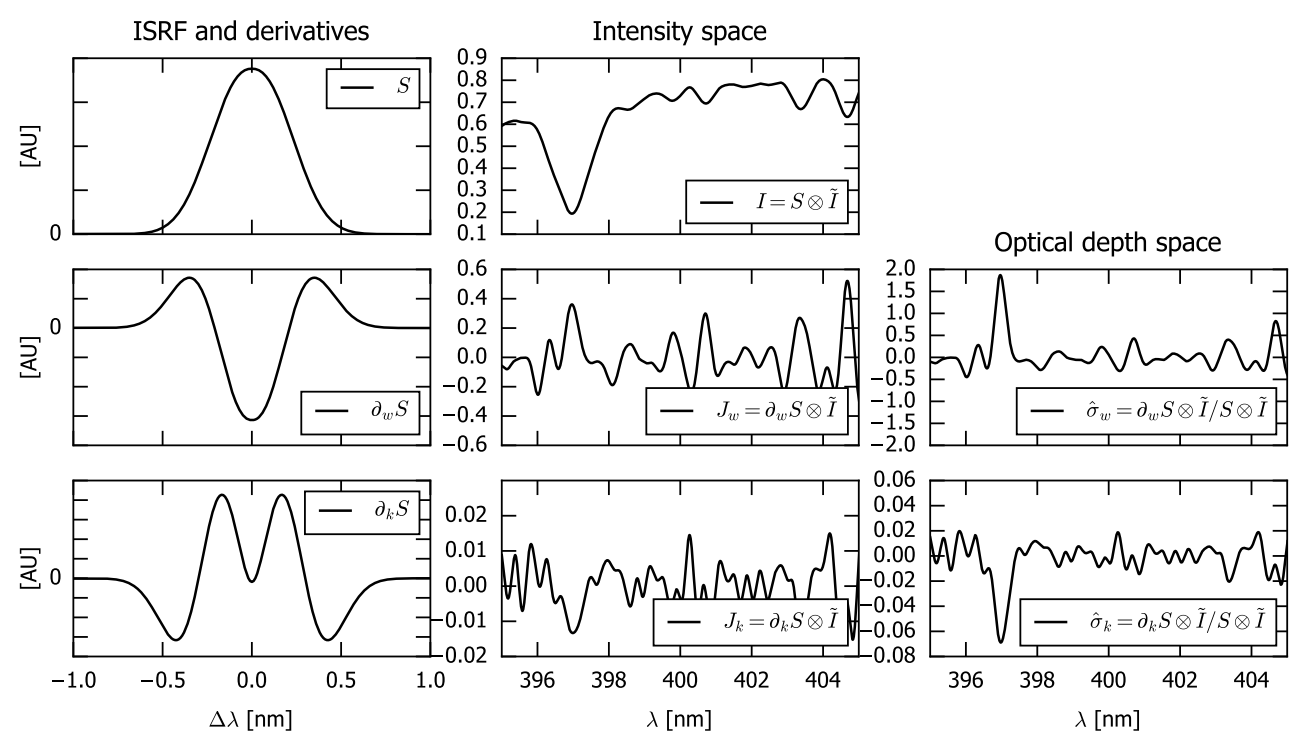

Figure 2. Illustration of the effect of ISRF changes on spectra in intensity and optical depth space. Left: super-Gaussian $S$ for $w=0.3 \mathrm{~nm}$ and $k=2.3$ (top) and its partial derivatives with respect to $w$ (center) and $k$ (bottom). Center: intensity $I$, derived from a high-resolution solar spectrum (Kurucz et al., 1984) by convolution with $S$ (top), and the RCS $J_{w}$ and $J_{k}$ caused by changes of $w$ (center) and $k$ (bottom), according to Eq. (12). Right: PA $\hat{\sigma}$ caused by changes of $w$ (center) and $k$ (bottom) in optical depth, according to Eq. (19). The actual change of OD results after scaling with $\Delta w$ (in nm) and $\Delta k$, respectively. For instance, a change of the ISRF width of $\Delta w=0.01 \mathrm{~nm}$ causes OD changes of about $5 \%$ up to $2 \%$ around the strong calcium line at $396.85 \mathrm{~nm}$.

denoting the partial derivative of $P$ with respect to $p$, evaluated at $p^{*}$. For illustration, Fig. 2 (left) displays $P=S$ and its partial derivatives with respect to $w$ and $k$.

Thus, the change of $P$ with respect to the baseline can be linearized:

$\Delta P:=P-P^{*} \approx \Delta p \times \partial_{p} P$.

The error made by neglecting the nonlinear $\mathcal{O}(2)$ terms is quantified in Appendix C. As rule of thumb, linearization works well for relative parameter changes below $10 \%$.

\subsubsection{Impact on $I$ : resolution correction spectra (RCS)}

The ISRF describes the response to a monochromatic input. For a high-resolution input spectrum $\widetilde{I}$, the measured signal $I$ results from the convolution of $\widetilde{I}$ with the ISRF:

$I=P \otimes \widetilde{I}$.

Consequently,

$$
\begin{aligned}
\Delta I & :=I-I^{*}=P \otimes \widetilde{I}-P^{*} \otimes \widetilde{I} \\
& =\Delta P \otimes \widetilde{I} \approx \Delta p \times \partial_{p} P \otimes \widetilde{I}=\Delta p \times J_{p}
\end{aligned}
$$

describes the effect of changes of $P$ on the measured spectrum $I$, expressed as the spectral structure

$J_{p}=\partial_{p} P \otimes \widetilde{I}$,

scaled by the parameter change $\Delta p$. Below, we refer to $J_{p}$ as RCS. Figure 2 (center column) displays $J_{w}$ and $J_{k}$ for a SG parameterization.
In case of a wavelength-dependent ISRF, $p$ can be approximated to change linearly around a central wavelength $\lambda_{\mathrm{c}}$, where $p=p_{\mathrm{c}}$ :

$p=p_{\mathrm{c}}+a \times\left(\lambda-\lambda_{\mathrm{c}}\right)$,

and thus

$\Delta p=a \times\left(\lambda-\lambda_{\mathrm{c}}\right)$.

Consequently, the RCS

$J_{p, \lambda}:=J_{p} \times\left(\lambda-\lambda_{\mathrm{c}}\right)$,

scaled by $a$ (i.e., the change of $p$ per wavelength), reflects the spectral structures caused by a linear wavelength dependency of parameter $p$. Wavelength dependencies of higher order $\left(\lambda-\lambda_{\mathrm{c}}\right)^{n}, n \geq 2$ can be parameterized analogously if necessary. This approach allows for a simple implementation of the wavelength dependency of the ISRF within wavelength calibration, as demonstrated in Sect. 5.2.

\subsubsection{Impact on convolved cross sections}

If RCS are included in the spectral calibration procedure (see Appendix B), the fit parameters of $J_{p}$ directly yield the change $\Delta p$ of the ISRF parameter $p$. This can be used for an improved convolution of absorption cross section $\widetilde{\sigma}$ :

$\sigma=\left(S+\Delta p \partial_{p} S\right) \otimes \widetilde{\sigma}$. 


\subsubsection{Impact on DOAS: pseudo-absorber}

Within DOAS analysis, slant column densities (SCDs) $s$, i.e., concentrations integrated along the effective light path, of atmospheric absorbers can be derived from the measured OD by solving

$\tau=-\ln \frac{I}{I_{0}}=\sum_{i} s_{i} \sigma_{i}+\Pi$,

where $\sigma_{i}$ is the absorption cross section of trace gas $i$, and $\Pi$ is a closure polynomial in wavelength accounting for Mie and Rayleigh scattering as well as other low-frequency contributions. It is common practice to account for other effects beyond actual trace gas absorptions in a formally analogue way by including PA $\hat{\sigma}_{i}$, i.e., spectral structures with physical meaning and units different from real absorption cross sections, but applicable in the same mathematical formalism. Commonly used PA are a "Ring spectrum", accounting for rotational Raman scattering, or an inverse intensity spectrum accounting for an intensity offset. Spectral shift and stretch can also be accounted for by PAs (Beirle et al., 2013).

Respective PAs can be defined in order to include the effects of ISRF changes in DOAS analysis in a linearized way: In optical depth space, the respective change caused by $\Delta p$ is

$\Delta \tau=\ln \left(I^{*}+\Delta I\right)-\ln \left(I^{*}\right)=\ln \left(1+\frac{\Delta I}{I^{*}}\right) \approx \frac{\Delta I}{I^{*}}$,

again illustrated for $S$ in Fig. 2 (right).

Thus, the PA is defined as

$\hat{\sigma}_{p}=\frac{\partial_{p} P \otimes \widetilde{I}}{P \otimes \widetilde{I}}$

for an overall change of $p$ (compare Fig. 2), and as

$\hat{\sigma}_{p, \lambda}=\frac{\partial_{p} P \otimes \widetilde{I}}{P \otimes \widetilde{I}} \times\left(\lambda-\lambda_{\mathrm{c}}\right)$,

for a wavelength-dependent change. Note that while RCSs are defined on high spectral resolution, PAs have to be sampled on the instrument's wavelength grid in order to be included in DOAS analysis. In case of ISRF derivatives which are not resolved by the instrument, the respective PAs are undersampled.

The respective factors $\hat{s}_{i}$ ("pseudo-SCDs") in Eq. (17) directly represent the change of the ISRF parameter $\Delta p$. By including the spectral patterns related to ISRF changes in DOAS analysis, fit quality improves (residuals are reduced), which generally reduces statistical as well as systematic errors of the derived trace gas SCDs, and is a prerequisite for the accurate retrieval of trace gases with low optical depths, such as glyoxal or HONO. In addition, the information on the ISRF change might be of interest in itself for diagnosis of the instrument's state.
The above formalism allows for a unique definition of PAs by Eqs. (19) and (20) if $\widetilde{I}$ is taken from a high-resolution solar atlas, such as provided by Kurucz et al. (1984). Thereby the effects of additional trace gas absorptions in the measured spectrum are neglected, which is justified as the spectral structures of all (direct or scattered) solar spectra are usually by far dominated by the Fraunhofer lines. However, in case of absorbers with high optical depth, e.g., for ozone in the UV or water vapor in the red spectral range, the effect of ISRF changes (and thus the appropriate PA) depend on the OD of the trace gas of interest. This might be accounted for by, e.g., calculating various PA for different a priori OD, and determine the appropriate PA matching the measurement iteratively.

In this section, the impact of ISRF changes is derived generally for any ISRF parameterization $P$. The SG $S$, however, is particularly suited for this approach due to the limited number of parameters, i.e., PAs, and the tangible meaning of the parameters $w$ (width) and $k$ (shape) and, optionally, $a_{w}$ (asymmetry). The same would hold for a parameterization based on a measured ISRF tuned by, e.g., widening or sharpening parameters as in Sun et al. (2016), which might be preferable when high-quality pre-launch measurements of the ISRF of satellite instruments are available and analytical parameterizations do not meet accuracy requirements.

\section{Datasets and instruments}

In this section, we briefly describe the datasets and instruments used in this study. Further details are provided in the given references.

\subsection{High-resolution solar atlas}

A solar spectrum with high accuracy and high spectral resolution is required for the calculation of RCS and PAs (previous section) and the wavelength calibration as described in Appendix B. For this purpose, we use the solar atlas provided by Kurucz et al. (1984).

In order to limit computational costs (e.g., for the convolution with the ISRF), the original data were pre-convolved with a Gaussian of $\sigma=0.025 \mathrm{~nm}$ width and sampled on a regular $0.01 \mathrm{~nm}$ grid. We found no indication for systematic effects on our results related to under-sampling. As the resulting spectrum $\widetilde{I}$ is used for all following convolutions within this study, the resulting widths $w$ are slightly biased low (as they miss the pre-convolution), but the effect is negligible.

\subsection{Avantes spectrometer}

We exemplarily illustrate the ISRF parameterization for a MAX-DOAS instrument based on an Avantes ultra-low stray-light spectrometer (AvaSpec-ULS2048x64) using a 
back-thinned Hamamatsu S11071-1106 detector. The instrument is similar to that described in Lampel et al. (2015).

The spectrometer is temperature stabilized $\left(\Delta T<0.02{ }^{\circ} \mathrm{C}\right)$. The UV spectrometer covered a spectral range of $296-459 \mathrm{~nm}$ at a FWHM spectral resolution of $\approx 0.55 \mathrm{~nm}$ (at $334 \mathrm{~nm}$ ) or $\approx 6$ pixel. The spectral stability was typically better than \pm 3 pm per day and better than $\pm 5 \mathrm{pm}$ for the duration of the measurements from 23 April 2015 until 3 March 2016 at the Penlee Point Atmospheric Observatory on the southwest coast of the UK (e.g., Yang et al., 2016). No significant change of the ISRF, measured each night based on an $\mathrm{Hg}$ discharge lamp, was observed during the campaign.

\subsection{GOME-2}

GOME-2 on MetOp-A was launched in 2006 as first of three GOME-2 instruments, providing a multi-annual time series of spectral measurements of the light reflected by the Earth's surface and atmosphere. The instrumental characteristics of GOME-2 are described in Callies et al. (2000) and Munro et al. (2016). The optical spectrometer covers the spectral range from 240 to $790 \mathrm{~nm}$ in four different channels. Spatial coverage is achieved by scanning the Earth via a scan mirror. The spectrometers have spectral resolutions (FWHM) of about $0.26 \mathrm{~nm}$ (UV) to $0.51 \mathrm{~nm}$ (VIS), with the ISRF usually parameterized by a Gaussian (Siddans et al., 2006; Munro et al., 2016).

GOME-2 spectral measurements are provided by EUMETSAT. In this study, we investigate the daily solar measurements from GOME-2 on MetOp-A for the years 20072014, and Earth's backscattered radiance for one arbitrarily chosen orbit on 1 April 2009.

\subsection{OMI}

OMI on AURA was launched in 2004 as part of the "A-train" (Levelt et al., 2006). It covers the spectral range from 270 to $500 \mathrm{~nm}$ in two spectral bands: the UV with a resolution of $\approx 0.42-0.45 \mathrm{~nm}$ FWHM and the VIS with a FWHM of about $0.63 \mathrm{~nm}$.

OMI is operated in push-broom mode, i.e., the across-track dimension is measured simultaneously by a CCD instead of scanned consecutively by a mirror, as for GOME-2. This implies that different viewing angles have different instrument properties, i.e., ISRFs.

For OMI, the ISRF is significantly different from a simple Gaussian, being more flat-topped (Dirksen et al., 2006). The operational parameterization of the OMI ISRF is thus composed of a Gaussian and a "broadened Gaussian", which corresponds to a SG with a fixed $k=4$.

In this study, we use the solar irradiance climatology compiled from daily OMI measurements in 2005.

\subsection{TROPOMI/Sentinel 5 Precursor}

TROPOMI will be launched in 2017 within the S-5P mission (Veefkind et al., 2012). Its instrumental design is similar to OMI, but TROPOMI provides higher spatial resolution and additional NIR and short-wave infrared (SWIR) channels. The UV, UVIS, and NIR spectral bands cover the spectral ranges 270-320, 320-490, and 710-775 nm, with spectral resolutions (FWHM) of $0.45-0.5,0.45-0.65$, and 0.34 $0.35 \mathrm{~nm}$, respectively (A. Ludewig, personal communication, 2016). The SWIR spectral band covers the spectral range 2305-2385 nm with a FWHM of $0.25 \mathrm{~nm}$ (Veefkind et al., 2012).

The TROPOMI ISRF has been extensively measured on ground before launch based on various SLSs. Generally it was found to be extremely flat-topped for the UV below $310 \mathrm{~nm}$, Gaussian to triangular for the UVIS $(310-500 \mathrm{~nm})$, flat-topped for the NIR, and slightly flat-topped for the SWIR.

Here we investigate the performance of the SG parameterization for sample TROPOMI ISRFs for each spectral channel. The respective calibration measurements for UV, UVIS, and NIR are based on a slit function stimulus (SFS) constructed by a monochromator using a rotating grating, and have been provided by Antje Ludewig and Joost Smeets from KNMI (personal communication, 2016). The SWIR calibration measurements were performed with an optical parametric oscillator (OPO) and have been provided by Paul Tol from SRON (personal communication, 2016).

\section{Parameterizing the ISRF by a super-Gaussian}

In this section we investigate the performance of a superGaussian parameterization of the ISRF for different detectors and demonstrate its benefits compared to a simple Gaussian parameterization.

\subsection{Avantes}

Figure 3 displays the $\mathrm{Hg}$ line at $404.66 \mathrm{~nm}$ (left) and a zenithsky spectrum (right) measured by the Avantes spectrometer described in Sect. 3.2 and the results of a least-squares fit of the ISRF parameterized as Gaussian, $G$ (green), or superGaussian, $S$ (orange). For the zenith-sky spectrum, the ISRF is fitted within the spectral calibration procedure, making use of the highly structured Fraunhofer lines (see Appendix B). The respective fit results are listed in Tables 1 and 2, and the residual of the wavelength calibration is shown in Fig. 3 (right bottom).

The simple Gaussian roughly reproduces the width of the measured $\mathrm{Hg}$ line (Fig. 3 left); however, $G$ cannot reflect the flat-topped shape. The spectral calibration of the measured spectrum converges (Fig. 3 right), but the resulting residual is rather large (5\% RMS). The fitted width $w$ is 0.336 and 

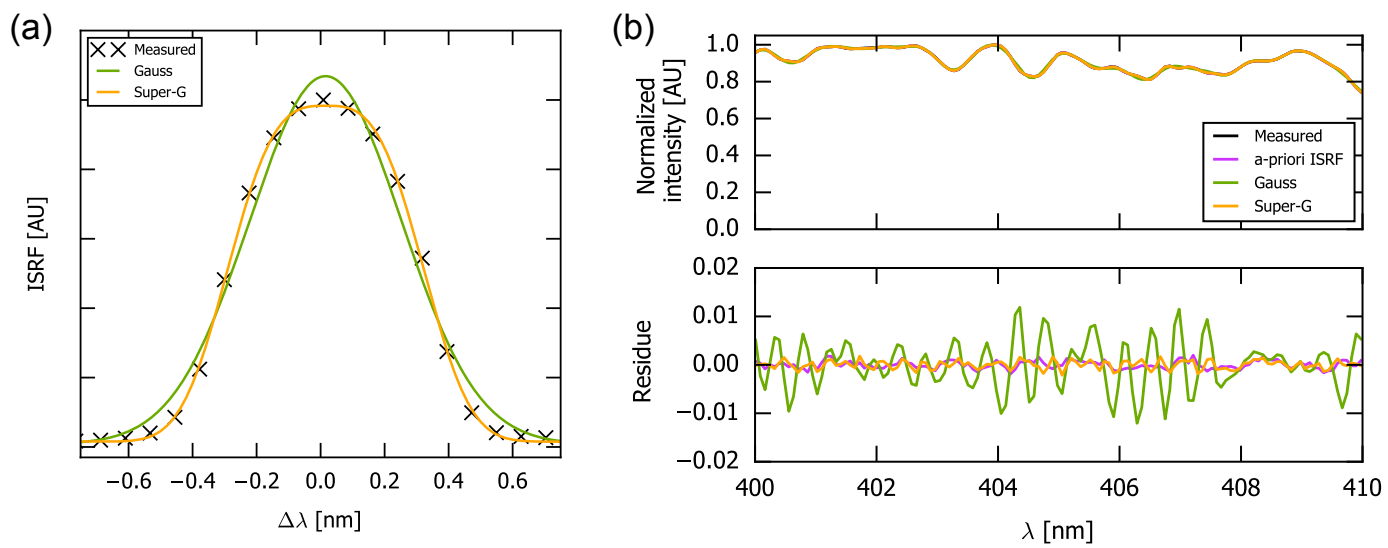

Figure 3. ISRF fit results for Avantes. Left: measured (crosses) line shape of the $\mathrm{Hg}$ line at $404.66 \mathrm{~nm}$ and best matching parameterizations $G$ and $S$. Right: least-squares fit of the ISRF during wavelength calibration of a zenith-sky measurement in the $400-410 \mathrm{~nm}$ interval (top) and corresponding residual (bottom), with ISRF derived from the $\mathrm{Hg}$ measurement ("a priori”) or parameterized as $G$ and $S$.

Table 1. Fit results of ISRF parameterized as $G$ or $S$ for the $\mathrm{Hg}$ line at $404.66 \mathrm{~nm}$ as measured by the Avantes spectrometer.

\begin{tabular}{lrrrr}
\hline $\begin{array}{l}\text { ISRF } \\
\text { model }\end{array}$ & $\begin{array}{r}\text { FWHM } \\
(\mathrm{nm})\end{array}$ & $\begin{array}{r}w \\
(\mathrm{~nm})\end{array}$ & $k$ & $\begin{array}{r}\text { RMS } \\
(\% o)^{*}\end{array}$ \\
\hline Gaussian & 0.560 & 0.336 & $\equiv 2$ & 31.10 \\
Super-G. & 0.620 & 0.348 & 3.15 & 6.77 \\
\hline
\end{tabular}

* Relative to maximum $\mathrm{Hg}$.

$0.353 \mathrm{~nm}$ for $\mathrm{Hg}$ fit and wavelength calibration, respectively, i.e., agrees within $5 \%$ for both fits.

The flat-topped shape of the measured $\mathrm{Hg}$ line is much better reflected by the super-Gaussian parameterization with a shape parameter $k=3.15$, and the spectral calibration results in much lower residuals $(0.884 \%$ o RMS for wavelength calibration). In addition, the performance of the wavelength calibration based on $S$ is almost as good as had the measured $\mathrm{Hg}$ line been taken as ISRF directly. Again, the fitted parameters of the direct ISRF fit and the wavelength calibration are consistent within $5 \%$ for both $w$ and $k$ (Tables 1 and 2).

The fitted $w$ (and thus the FWEM) for $G$ vs. $S$ are comparable within $5 \%$ as well. In contrast, the FWHM of the fitted ISRFs to the $\mathrm{Hg}$ line differ by more than $10 \%$ between $G$ and $S$ (Table 1). The concept of FWHM, widely used due to historic reasons when distribution widths were determined graphically, thus seems to be a suboptimal measure for the width of the ISRF for non-Gaussian shapes. We thus focus on $w(=1 / 2$ FWEM $)$ instead of FWHM hereafter.

\subsection{GOME-2}

For GOME-2, the ISRF is usually parameterized by a Gaussian (Siddans et al., 2006; Munro et al., 2016) or an asymmetric Gaussian (e.g., De Smedt et al., 2012). We have tested the benefit of a super-Gaussian parameterization over a Gaus-
Table 2. Fit results of ISRF parameterized as $G$ or $S$ for the wavelength calibration $(400-410 \mathrm{~nm})$ of the zenith-sky spectrum measured by the Avantes spectrometer.

\begin{tabular}{lrrr}
\hline $\begin{array}{l}\text { ISRF } \\
\text { model }\end{array}$ & $\begin{array}{r}w \\
(\mathrm{~nm})\end{array}$ & $k$ & $\begin{array}{l}\text { RMS } \\
(\% \circ)^{\mathrm{a}}\end{array}$ \\
\hline A priori $^{\mathrm{b}}$ & & & 0.81 \\
Gaussian & 0.353 & $\equiv 2$ & 5.00 \\
Super-G. & 0.339 & 3.28 & 0.88 \\
\hline a Relative to maximum intensity. ${ }^{\mathrm{b}}$ Measured \\
Hg line, offset corrected and interpolated.
\end{tabular}

sian, both symmetric as well as asymmetric, exemplarily for a direct sun measurement on 23 January 2007. For the asymmetric parameterizations as defined in Eq. (A1), the asymmetry parameter $a_{w}$ is included, allowing for different widths on both flanks of the ISRF (see Appendix A).

Figure 4 (left) displays the measured sun spectrum (black) and the results of the wavelength calibration for asymmetric $G$ and $S$ in the interval $420-440 \mathrm{~nm}$. This wavelength range was chosen for comparison of the long-time evolution of the ISRF with Munro et al. (2016); see Sect. 5.1.1. The fitted asymmetric ISRFs are displayed in Fig. 4. For comparison, the operational ISRF from GOME-2 key data is included as well. Fit results (including symmetric parameterizations) are summarized in Table 3.

Symmetric and asymmetric parameterizations yield basically the same results, and the fitted asymmetry parameters are close to zero. But still, allowing for asymmetry significantly improves the fit quality (this effect is much larger for the UV spectral range). For the asymmetric parameterization, the fitted widths $w$ are $0.301 \mathrm{~nm}$ for $G$ and $0.307 \mathrm{~nm}$ for $S$. The shape parameter $k$ for the fitted $S$ was found to be 2.17 . The super-Gaussian is thus close to a simple Gaussian, and the benefit of $S$ over $G$ is far less significant than for the 
(a)

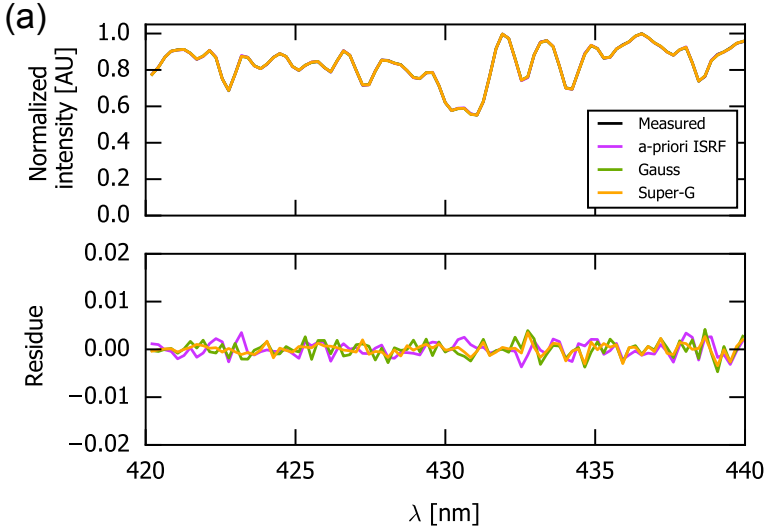

(b)

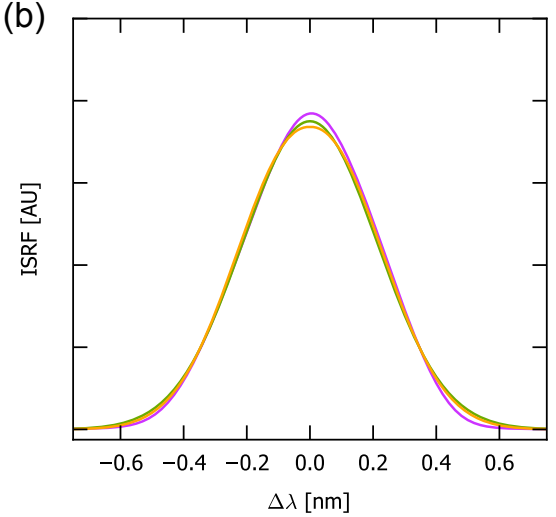

Figure 4. ISRF fit results for the GOME-2 direct sun measurement on 23 January 2007. Left: least-squares fit of the ISRF during wavelength calibration in the $420-440 \mathrm{~nm}$ interval (top) and corresponding residual (bottom), with ISRF parameterized as $G$ and $S$ and the official ISRF (purple). Right: illustration of the best matching ISRF parameterizations $G$ and $S$ and the official ISRF (purple).

Table 3. Fit results of ISRF parameterized as $G$ or $S$ for the wavelength calibration $(420-440 \mathrm{~nm})$ of the direct solar spectrum measured by GOME-2.

\begin{tabular}{lrrrr}
\hline $\begin{array}{l}\text { ISRF } \\
\text { model }\end{array}$ & $\begin{array}{r}w \\
(\mathrm{~nm})\end{array}$ & $k$ & $\begin{array}{r}a_{w} \\
(\mathrm{~nm})\end{array}$ & $\begin{array}{r}\mathrm{RMS} \\
(\% \circ)^{*}\end{array}$ \\
\hline A priori & & & & 1.51 \\
Gaussian & 0.301 & $\equiv 2$ & $\equiv 0$ & 1.62 \\
Super-G. & 0.307 & 2.18 & $\equiv 0$ & 1.18 \\
Asym. Gaussian & 0.301 & $\equiv 2$ & -0.012 & 1.46 \\
Asym. super-G. & 0.306 & 2.17 & -0.010 & 1.02 \\
\hline
\end{tabular}

* Relative to maximum intensity.

Avantes spectrometer (previous section) or OMI (next section). For the fit shown in Fig. 4, the use of an asymmetric super-Gaussian parameterization within wavelength calibration improves the fit RMS to $1.02 \%$, compared to 1.46 and $1.51 \%$ for an asymmetric Gaussian parameterization and the ISRF from key data, respectively.

\subsection{OMI}

Figure 5 displays the wavelength calibration for the OMI sun climatology based on the official ISRF (at $430 \mathrm{~nm}$ ) and parameterization $G$ and $S$. Fit results are summarized in Table 4 .

Obviously, a parameterization of the ISRF by a Gaussian is not appropriate for OMI and results in a highly structured residual with 5.64\% RMS. With the super-Gaussian parameterization, residuals are significantly smaller $(0.85 \%$ o RMS $)$.

The operational OMI ISRF has been found to be asymmetric (Dirksen et al., 2006). However, for the asymmetric parameterization, $a_{w}$ was found to be very small $(-0.005 \mathrm{~nm})$, the fitted ISRF hardly changes, and the fit residual hardly improves over the symmetric $S$ (see Table 4). The fit results
Table 4. Fit results of ISRF parameterized as $G$ or $S$ for the wavelength calibration $(420-440 \mathrm{~nm})$ of the direct solar spectrum measured by OMI.

\begin{tabular}{lrrrr}
\hline $\begin{array}{l}\text { ISRF } \\
\text { model }\end{array}$ & $\begin{array}{r}w \\
(\mathrm{~nm})\end{array}$ & $k$ & $\begin{array}{r}a_{w} \\
(\mathrm{~nm})\end{array}$ & $\begin{array}{r}\mathrm{RMS} \\
(\% \circ)^{*}\end{array}$ \\
\hline A priori & & & & 2.29 \\
Gaussian & 0.336 & $\equiv 2$ & $\equiv 0$ & 5.64 \\
Super-G. & 0.362 & 3.44 & $\equiv 0$ & 0.85 \\
Asym. SG & 0.362 & 3.44 & -0.004 & 0.84 \\
\hline
\end{tabular}

* Relative to maximum intensity.

for $S$ are still better (in terms of RMS) than those derived based on the operational ISRF ("a priori") derived from preflight measurements. This might indicate a slight change of the ISRF after launch. However, the shape of a priori and SG ISRFs (in particular the flanks) is quite similar (see Fig. 5b).

\subsection{TROPOMI/S-5P}

We apply the super-Gaussian parameterization exemplarily to one set of SFS measurements for each TROPOMI detector around the center row and column of the detector, corresponding to the central wavelength and nadir-viewing geometry. The ISRFs and best matching parameterizations $G$ and $S$ are illustrated in Fig. 6. Note that at the detector center, TROPOMI ISRFs are quite symmetric. In Appendix A, also asymmetric samples from the detector edges are shown.

The TROPOMI ISRF is different for the four detectors. In the UVIS, it is similar to a Gaussian, while it is more flattopped in the NIR and almost approaching a boxcar shape in the UV. However, the SG parameterization is capable of reproducing the measured ISRFs well with shape parameters of 7.4, 2.4, 3.0, and 2.7 for the UV, UVIS, NIR, and SWIR, respectively. 

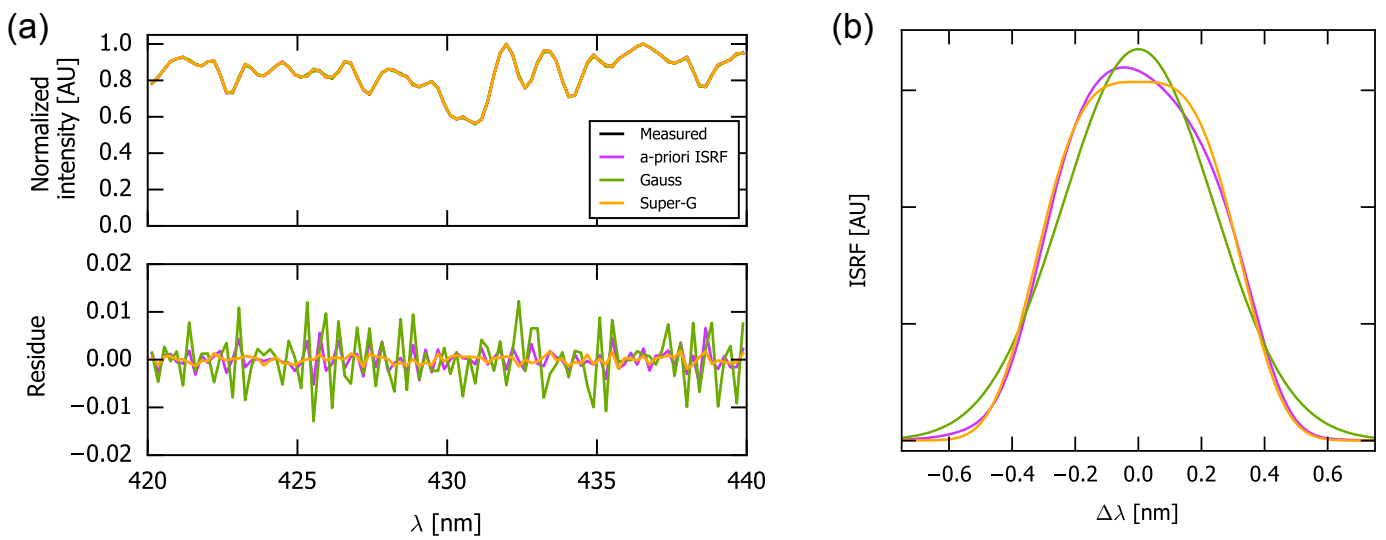

Figure 5. ISRF fit results for the OMI direct sun measurement climatology for cross-track pixel 2 (0-based). Left: least-squares fit of the ISRF during wavelength calibration in the $420-440 \mathrm{~nm}$ interval (top) and corresponding residual (bottom), with ISRF parameterized as $G$ and $S$ and the official ISRF (purple). Right: illustration of the best matching ISRF parameterizations $G$ and $S$ and the official ISRF (purple).
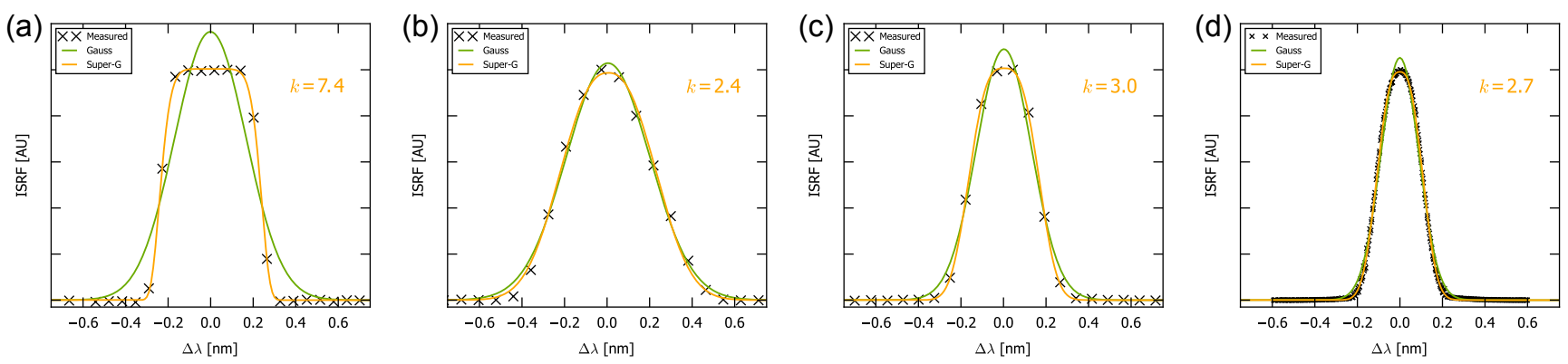

Figure 6. Exemplary TROPOMI ISRF for the UV (a), UVIS (b), NIR (c), and SWIR (d) at the detector centers. Crosses indicate the prelaunch measurements (provided by Antje Ludewig (UV, UVIS, NIR) and Paul Tol (SWIR), personal communication, 2016). Lines show the best matching parameterizations $G$ and $S$. The respective fit parameters are listed in Table 5.

Table 5. Fit results of ISRF parameterized as $G$ or $S$ for sample TROPOMI pre-launch calibration measurements at detector center.

\begin{tabular}{llrrr}
\hline Detector & $\begin{array}{l}\text { ISRF } \\
\text { model }\end{array}$ & $\begin{array}{r}w \\
(\mathrm{~nm})\end{array}$ & $k$ & $\begin{array}{r}\text { RMS } \\
(\%))^{*}\end{array}$ \\
\hline UV & Gaussian & 0.237 & $\equiv 2$ & 124.1 \\
& Super-G. & 0.246 & 7.38 & 7.5 \\
\hline UVIS & Gaussian & 0.277 & $\equiv 2$ & 21.1 \\
& Super-G. & 0.284 & 2.39 & 15.4 \\
\hline \multirow{2}{*}{ NIR } & Gaussian & 0.183 & $\equiv 2$ & 28.2 \\
& Super-G. & 0.191 & 3.03 & 4.2 \\
\hline \multirow{2}{*}{ SWIR } & Gaussian & 0.126 & $\equiv 2$ & 25.4 \\
& Super-G. & 0.130 & 2.69 & 5.9 \\
\hline
\end{tabular}

* Relative to maximum intensity.
The official ISRF parameterizations (Antje Ludewig and Paul Tol, personal communication, 2016) are based on

- advanced sigmoids, involving nine parameters, for the UV and NIR;

- a "generalized exponential" with eight parameters for the UVIS; and

- the convolution of a skew-normal and a block distribution, plus a Pearson VII distribution, for the SWIR, involving eight parameters in total.

These customized parameterizations allow for a very accurate fit of the ISRF to SFS and OPO measurements. From our experience, however, it will not be possible to use a parameterization with so many (correlated) parameters within the wavelength calibration procedure. In contrast, the SG parameterization is expected to work well within wavelength calibration due to the low number of (uncorrelated) parameters, as demonstrated for OMI, and the fitted SG is expected to be applicable for DOAS-like applications in the UV/UVIS. However, the fit performance of the SG param- 
eterization might not be sufficient for applications with high accuracy requirements.

\section{Parameterizing changes of the ISRF by RCS and PA}

In the second part of the paper we present applications of the linearisation of ISRF changes derived in Sect. 2.2. As stated therein, this concept might be applied to any ISRF parameterization, but the SG is particularly useful due to the low number of parameters and their illustrative meaning.

In Sect. 5.1 we investigate changes of the ISRF over time, i.e., long-term as well as in-orbit changes, exemplarily for GOME-2. ${ }^{2}$

In Sect. 5.2 we demonstrate the concept of considering the wavelength dependency of the ISRF by RCS.

\subsection{Changes of the ISRF over time}

\subsubsection{Long-term changes of the GOME-2 ISRF}

Munro et al. (2016) have shown that the GOME-2 ISRF changes over time, in particular for the UV and blue spectral range. Figure 10 in Munro et al. (2016) shows that the FWHM of a Gaussian fitted to SLS measurements during daily calibration decreases by about $5 \%$ from 2007 to 2010 at $429 \mathrm{~nm}$. In addition, the FWHM reveals a seasonal pattern. Munro et al. (2016) have related this temporal pattern of the GOME-2 ISRF to the optical bench temperature and found good correlation.

We investigate the temporal evolution of the GOME-2 ISRF width around $429 \mathrm{~nm}$ by performing wavelength calibration fits for the daily solar spectra for four different fit settings:

1. The ISRF is fitted as Gaussian, as in Munro et al. (2016).

2. The ISRF is fitted as super-Gaussian.

3. The ISRF is fixed to the results of setting 2 for the first day of the time series.

4. As in setting 3 but, in addition, the RCS $J_{w}$ and $J_{k}$, derived from Taylor expansion, are included in the fit (see Eq. 12).

Figure 7 displays the time series of fit results for $w, k$, and the fit RMS (relative to the Gaussian fit).

1. The first evaluation reproduces the findings shown in Munro et al. (2016): the ISRF width decreases from 2007 to the end of 2009, when the detector was heated during throughput tests. Afterwards, seasonal variations dominate on top of a constant level. Note that the time series shown in Fig. 7 and in Fig. 10 in Munro et al.

\footnotetext{
${ }^{2}$ For OMI (not shown), we could not find indications for a significant change of the ISRF over time.
}

(a)

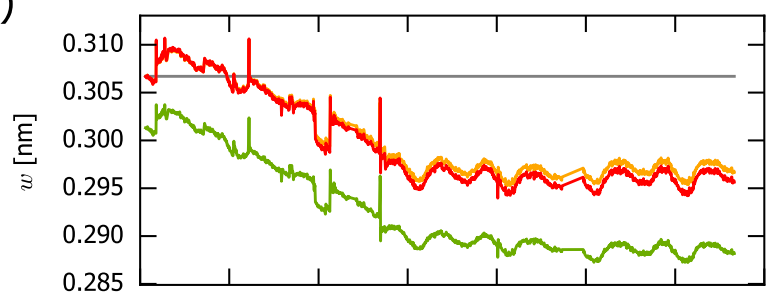

(b)

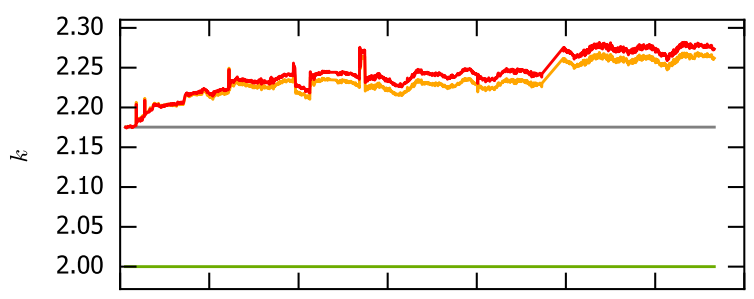

(c)

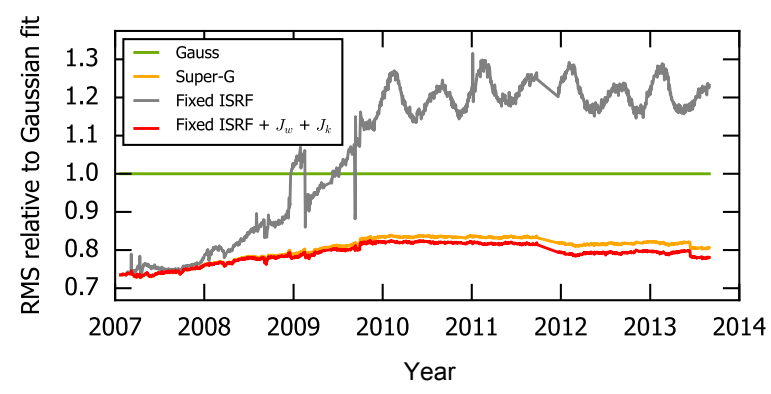

Figure 7. Temporal evolution of the GOME-2 ISRF fitted for daily solar measurements $(420-440 \mathrm{~nm})$ for four fit settings, i.e., a Gaussian parameterization (green), a super-Gaussian parameterization (orange), a fixed ISRF matching the super-Gaussian from the first day (grey), and a fixed ISRF plus the RCS $J_{w}$ and $J_{k}$ (red). The subplots display the fitted width (a), shape (b), and fit RMS relative to the Gaussian fit (c).

(2016) agree well within a surprising level of detail, though the latter was derived from calibration measurements, while the first is based on solar measurements.

2. The results of the SG fit have already been discussed in Sect. 4.2 for the first day of the time series: the SG slightly improves the fit residual and yields a shape parameter slightly above $2(k=2.17)$. The temporal evolution of $w$ is similar to that for the Gaussian fit, but shifted by about $0.005 \mathrm{~nm}$. Interestingly, the fitted $k$ also shows a clear temporal pattern, increasing by about 0.1 over the time series; i.e., not only the width, but also the shape of the GOME-2 ISRF has changed.

3. If wavelength calibration and ISRF fit are done in the beginning of the time series and the ISRF is kept constant afterwards, the resulting fit residual is almost as good as for setting 2 within 2007 but starts to increase significantly later on. In 2010, when the change of $w$ compared to the beginning of the time series reaches its maximum, the RMS increased by up to $50 \%$. 
4. In setting 4, the ISRF is kept constant as well, as for setting 3 , but the effect of ISRF changes is accounted for by including the RCS $J_{w}$ and $J_{k}$ in the wavelength calibration procedure. Time-dependent values for $w$ and $k$ are thus derived from the values of the a priori ISRF plus the respective RCS fit coefficients. Resulting $w$ and $k$ agree very well with setting 2 , and the fit RMS for setting 4 is even lower than for setting 2 . The explanation for this, at first glance unexpected, finding is that the GOME-2 ISRF, though well approximated by $S$, is not an exact super-Gaussian. The fit settings 2 and 4 span two slightly different groups of ISRF shapes, and setting 4 is slightly better representing the actual GOME-2 ISRF.

Thus, while the application of a fixed ISRF for the GOME2 time series begins to become suboptimal after 2 years, the additional inclusion of RCS actually accounts for the spectral changes caused by the ISRF changes over time.

\subsubsection{In-orbit changes}

The case study shown above illustrates that the linearisation of ISRF changes generally works; however, a full ISRF fit might easily be performed for each daily measured sun reference instead. This is different if the ISRF changes along orbit: due to the high number of spectra, a full fit of the ISRF is not feasible any more. Thus, in the case of trace gas retrievals, the concept of linearisation by accounting for changes by a PA, which can be included in a linear fit setup, is highly beneficial.

We have investigated the benefit of the PA $\hat{\sigma}_{w}$ for a sample fit in the visible spectral range for one orbit measured by GOME-2 A. Figure 8a displays the fit parameter $\Delta w$, which directly reflects the in-orbit change of the ISRF width $w$. A similar effect has been shown by Azam and Richter (2015, Fig. 23 therein), who derived the ISRF for each individual satellite pixel by a nonlinear fit of the solar atlas.

The systematic change of ISRF width along orbit is closely related to the temperature of the pre-disperser prism (Fig. 8a). Thus, the fit parameter of the fitted PA $\hat{\sigma}_{w}$ directly serves as a diagnostic tool for the instrument's state.

The respective change of the fitted shape parameter $k$ is shown in Fig. 8b. While significantly increasing, the effect is negligibly small.

Figure 8c displays the fit RMS for retrievals with and without both $\hat{\sigma}_{w}$ and $\hat{\sigma}_{k}$. The inclusion of the PA significantly improves the fit and removes a systematic component of the residual, which is a prerequisite for improved trace gas analyses, in particular for trace gases with low OD.

\subsection{Changes of ISRF over wavelength}

The ISRF generally depends on wavelength. In Sect. 2.2, it is shown that the spectral structure caused by ISRF changes over wavelength can be linearized as well. In this
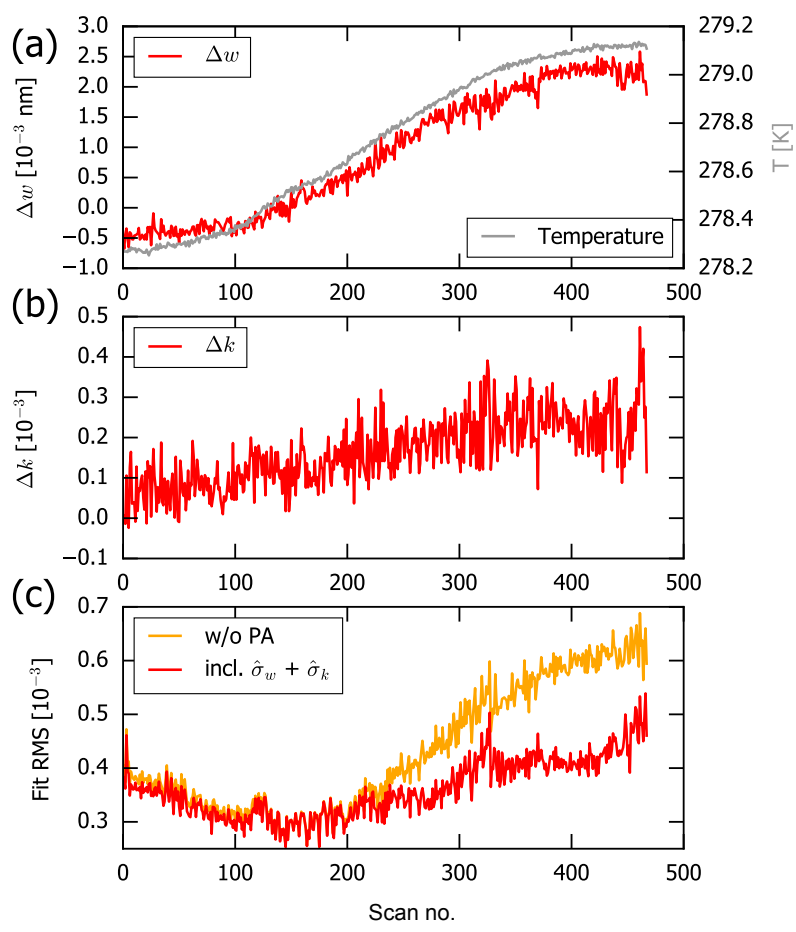

Figure 8. Temporal change of the GOME-2 ISRF along orbit on 1 April 2009 as derived from a linear fit including the PAs $\hat{\sigma}_{w}$ and $\hat{\sigma}_{k}$. Top: in red, the fit results for $\Delta w$ are shown, indicating the change of the ISRF width. Fit results are averaged over one full GOME- 2 scan (24 forward and 8 backscan pixels). In grey, the temperature of the predisperser prism, as provided in the operational lv2 files, is shown. Center: fit results for $\Delta k$ averaged over one scan. Bottom: fit RMS (based on residuals averaged over one scan) compared for retrievals with and without PA.

section, we demonstrate this concept for a synthetic spectrum (Sect. 5.2.1) as well as actual GOME-2 measurements (Sect. 5.2.2).

\subsubsection{Proof of concept: synthetic spectrum}

We construct a synthetic spectrum by convolution of $\widetilde{I}$ with a wavelength-dependent ISRF with $w$ increasing linearly by $0.003 \mathrm{~nm} \mathrm{~nm}^{-1}$, i.e., from $0.27 \mathrm{~nm}$ (at $420 \mathrm{~nm}$ ) to $0.33 \mathrm{~nm}$ (at $440 \mathrm{~nm}$ ). The ISRFs and the resulting spectrum are illustrated in Fig. 9.

In Fig. 10, the wavelength calibration results are shown for (a) a simple SG parameterization with wavelengthindependent ISRF (orange) and (b) additional inclusion of the RCS $J_{w, \lambda}=J_{w} \times\left(\lambda-\lambda_{\mathrm{c}}\right)$.

The fit of a wavelength-independent super-Gaussian yields the average $w$ correctly and results in small residuals at the fit window center, where the actual width matches the average. However, towards the edges of the fit window, the residual increases systematically due to the linear change of the true ISRF width. Overall RMS is $2.34 \%$. 

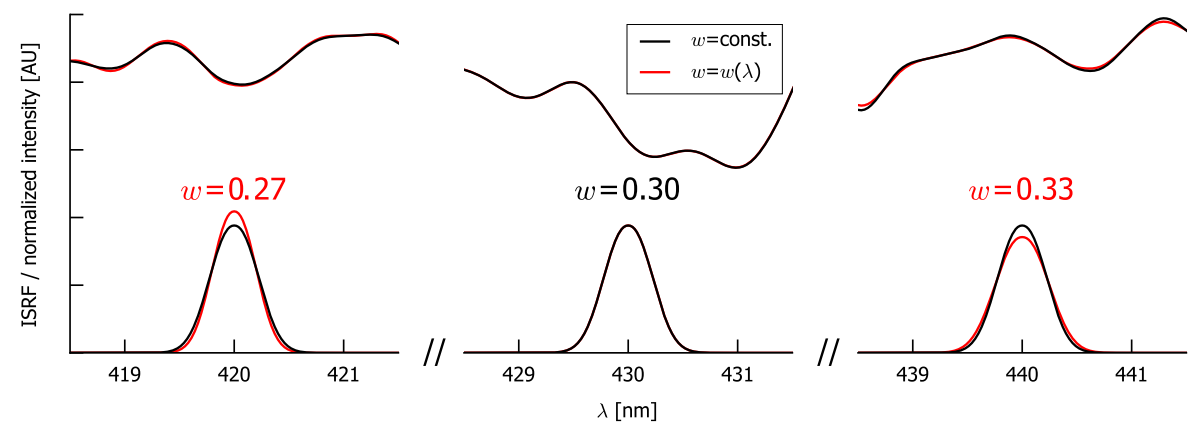

Figure 9. Illustration of the generation of a synthetic spectrum in order to investigate wavelength-dependent ISRF changes. In black, the result of $I=S \otimes \widetilde{I}$, based on the ISRF $S(w=0.3, k=2.2)$, is shown. In red, the respective $I$ for the wavelength-dependent ISRF is shown with $\Delta w=0.03 \mathrm{~nm} \mathrm{~nm}^{-1}$.
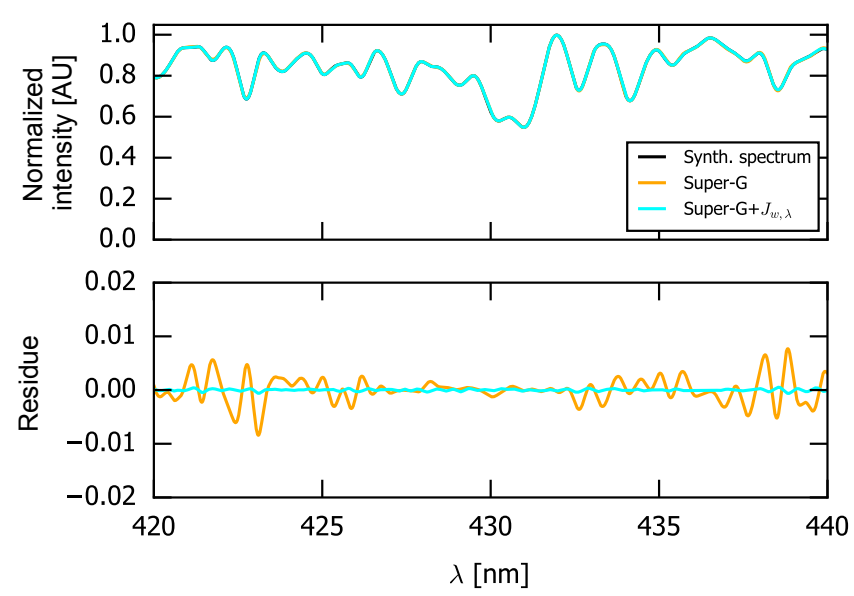

Figure 10. Fit results for the synthetic spectrum based on an ISRF fit with the spectral structure $J_{w, \lambda}$ excluded (orange) and included (cyan).

If the $\operatorname{RCS} J_{w, \lambda}$ is included in the fit, the synthetic spectrum can be reproduced almost perfectly with a fit RMS of $0.18 \%$, and the fitted change of $w$ over wavelength $\left(0.00297 \mathrm{~nm} \mathrm{~nm}^{-1}\right)$ is very close to the a priori $\left(0.003 \mathrm{~nm} \mathrm{~nm}^{-1}\right)$.

\subsubsection{Application: GOME-2}

In this section, we apply the concept of RCS for describing the ISRF wavelength dependency for GOME-2 measurements in the UV. We have determined the wavelength dependency of the ISRF, parameterized as $S_{a_{w}}$, by performing wavelength calibrations in small $(10 \mathrm{~nm}$ wide) fit windows ("subwindows") in steps of $5 \mathrm{~nm}$. A similar procedure is used in QDOAS (Danckaert et al., 2015) in order to determine wavelength dependencies of ISRF width and spectral shifts. Figure 11 displays the resulting parameters $w, k$, and $a_{w}$ as derived for the solar irradiance measured on 23 January 2007. The ISRF width of GOME-2 in the UV is generally decreasing with wavelength, the shape is approximately Gaus-
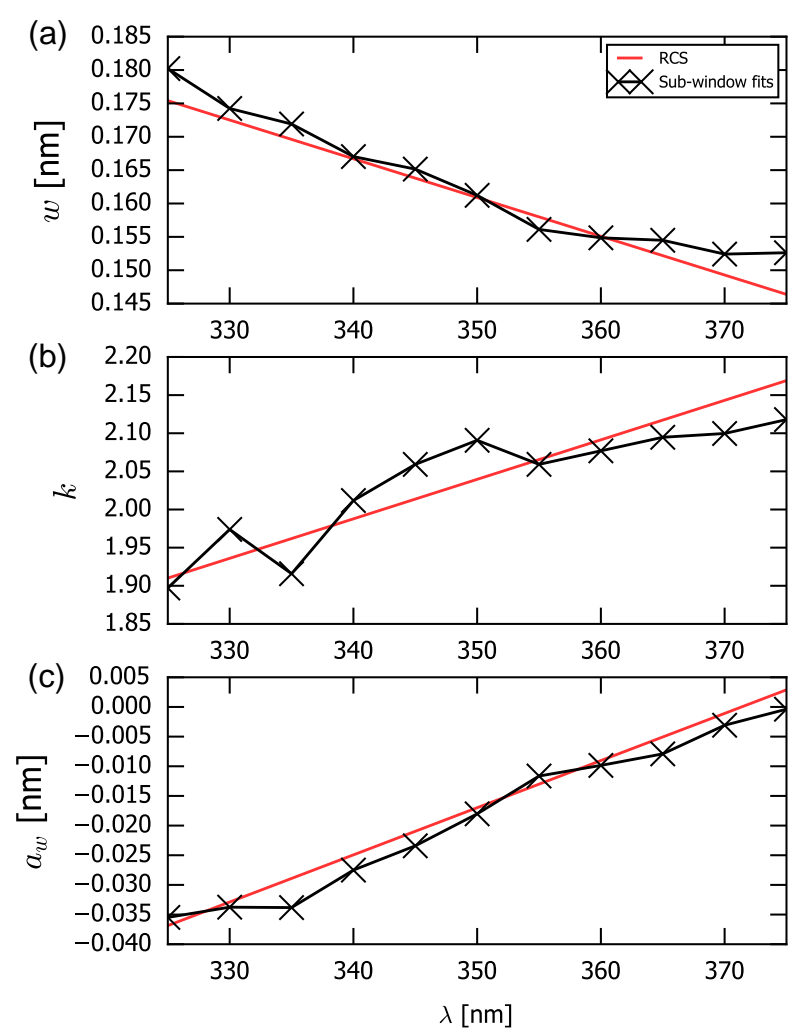

Figure 11. Wavelength dependency of the GOME-2 ISRF width $w$ (a), shape $k$ (b), and asymmetry $a_{w}$ (c), as derived from SG fits in subwindows of $10 \mathrm{~nm}$ width, sampled in $5 \mathrm{~nm}$ steps (black), and from the fit factors of the respective $\operatorname{RCS} J_{w, \lambda}, J_{k, \lambda}$, and $J_{a_{w}, \lambda}$ (red).

$\operatorname{sian}(k \approx 2)$ with increasing $k$, and the asymmetry parameter is negative for low wavelengths (meaning that the left flank of the ISRF is less steep than the right flank), increasing towards 0 (symmetry) at $375 \mathrm{~nm}$.

In a second step, we have performed the wavelength calibration over the full fit window (325-375 nm) at once, with wavelength dependencies accounted for by including the 
$\operatorname{RCS} J_{w, \lambda}, J_{k, \lambda}$, and $J_{a_{w}, \lambda}$. The respective wavelength dependency of $w, k$, and $a_{w}$ as determined by the RCS fit coefficients is included in Fig. 11 in red, showing generally good agreement to the subwindow fit results.

Figure 12 displays the respective fit of the solar irradiance, again for fit settings excluding or including RCS. As for the synthetic case study, including RCS significantly improves the fit results, particularly at the edges of the fit window.

Thus, the wavelength dependency of the ISRF can be accounted for by including RCS in the wavelength calibration procedure, while the actual convolution $S \otimes \widetilde{I}$ is done for a constant ISRF, which is by far faster and more stable than actually fitting a wavelength-dependent ISRF.

\section{Conclusions}

The super-Gaussian is a powerful extension of the Gaussian which allows to represent a variety of different shapes by adding just one free shape parameter $k$, in addition to $w$ describing its width. Optionally, asymmetry can be described by a further asymmetry parameter $a_{w}$. The super-Gaussian is particularly well suited for describing flat-topped ISRFs, as occur for OMI or TROPOMI (UV). Due to the low number of parameters, which are uncorrelated, the SG can be fitted within wavelength calibration of measurements of direct or scattered sunlight, making use of the highly structured Fraunhofer lines, which is generally challenging for sophisticated ISRF parameterizations with many parameters.

Changes of the ISRF over time or wavelength can be accounted for by including spectral structures derived from the linear term of a Taylor expansion. In intensity and OD space, RCS and PAs are defined to be included in spectral calibration and DOAS analysis, respectively. The linearization makes the spectral analysis robust and fast; thus the inclusion of RCS and PA comes without notable performance loss. While this approach is possible for any ISRF parameterization, the SG is particularly suited due to the low number of parameters and the illustrative meaning of its parameters.

For GOME-2, the inclusion of PAs significantly improves the fit quality and removes a systematic component of the residual along orbit, as it appropriately accounts for the effects of ISRF broadening along orbit. The fitted change of ISRF width directly corresponds to temperature. Generally, including RCS and PAs allows for easy monitoring of the long-term stability of an instrument by straightforward fit parameters.

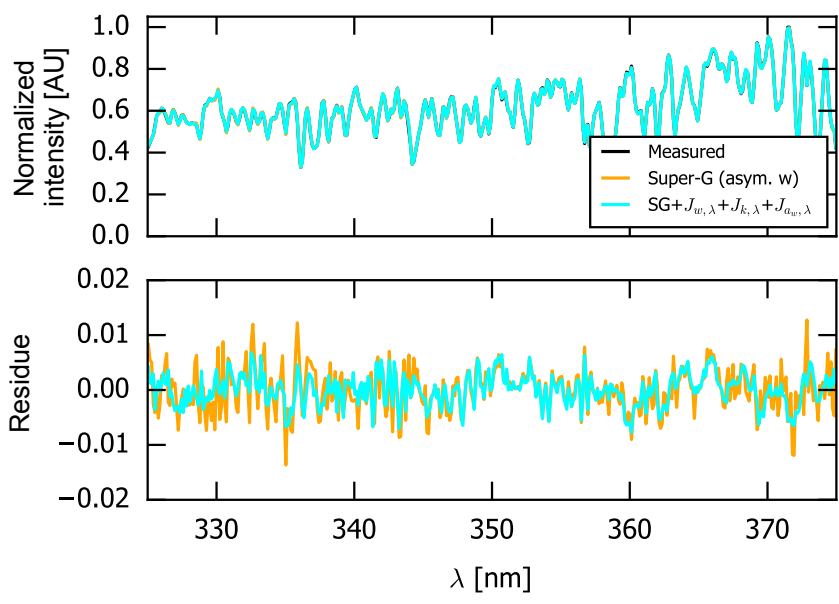

Figure 12. Fit result of the ISRF excluding (orange) or including (cyan) RCS for the solar spectrum of GOME-2 on 23 January 2007 in the UV.

Accounting for the wavelength dependency of the ISRF by the proposed linearisation allows for considering wide fitting windows during spectral calibration and is thus a fast and robust alternative for the "subwindow" approach as implemented in QDOAS (Danckaert et al., 2015) or fitting a polynomial for $w(\lambda)$ as in DOASIS (Lehmann et al., 2008).

\section{Data availability}

The solar atlas (Kurucz et al., 1984) is available at http://kurucz.harvard.edu/sun/fluxatlas/. The measurements of the Avantes spectrometer are provided in the Supplement. GOME-2 spectral measurements are provided by EUMETSAT and can be ordered at http://www.eumetsat.int/website/ home/Data/DataDelivery/DataRegistration/index.html.

OMI spectral measurements are provided by NASA at https://disc.gsfc.nasa.gov/Aura/data-holdings/OMI/. For TROPOMI ISRF measurements please contact Antje Ludewig (antje.ludewig@knmi.nl) and Paul Tol (P.J.J.Tol@sron.nl) for UV/UVIS/NIR and SWIR, respectively. 
Appendix A: Implementation of the asymmetric super-Gaussian

The super-Gaussian as defined in Eq. (2) is symmetric in $x$, while an ISRF might generally be asymmetric. For the case of a Gaussian, several implementations of asymmetric shapes have been proposed which basically use different values for $\sigma$ of the left and the right wing. Here, we follow a similar approach in order to allow for ASGs:

$S_{\text {asym }}(x)=A_{\text {asym }} \times\left\{\begin{array}{l}\exp \left(-\left|\frac{x}{w-a_{w}}\right|^{k-a_{k}}\right) \text { for } x \leq 0 \\ \exp \left(-\left|\frac{x}{w+a_{w}}\right|^{k+a_{k}}\right) \text { for } x>0\end{array}\right.$,

with the additional asymmetry parameters $a_{w}$ and $a_{k}$. For $a_{w}=a_{k}=0$, this becomes Eq. (2). For $a_{k}=0$, i.e., an ASG with asymmetric width, but symmetric shape parameter, we define

$S_{a_{w}}:=S_{\text {asym }, a_{k}=0}$.

Figure A1 displays examples of the ASG for different parameter settings.

Note that by this implementation of asymmetry, the FWEM of $S$ still equals $2 w$ independent of all other parameters (as opposed to, e.g., a parameterization based on two width parameters $w_{\text {left }}$ and $\left.w_{\text {right }}\right)$. This aspect is more than a sophisticated detail, as it implies that the ASG parameters are almost uncorrelated and allows for a multi-step procedure: within a first step, $w$ and $k$ might be estimated from a SG fit; in a second step, the asymmetry parameters can be optimized, while the values of $w$ and $k$ from the first step hardly change.

For an asymmetric function, the first moment ("center of mass", COM) is generally not 0 any more. Consequently, the application of such an asymmetric ISRF would cause a net spectral shift in the measured spectrum. However, the effect of a possible spectral shift is usually accounted for during spectral calibration and should not interfere with the asymmetry of the ISRF. In order to separate both effects, we demand that the ISRF does not cause a shift; i.e., after calculating the ASG according to Eq. (A1), the COM is determined, and the ASG is shifted accordingly and normalized to an integral of 1. Figure A2 shows the ISRFs resulting from the shifted ASG shown in Fig. A1.

In Fig. A3, sample ISRFs from the different TROPOMI detectors and fitted ASG are shown with varying level of asymmetry. Table A1 lists the respective fit results.

The combined variation of $a_{k}$ and $a_{w}$ can lead to quite exotic shapes. For some instruments (in particular some MiniMAX DOAS instruments, or the sample TROPOMI ISRF in the SWIR), this helped to slightly improve the fit performance for a direct ISRF measurement; however, within spectral calibration, the additional variety of possible shapes introduced by $a_{k}$ often results in unstable and diverging fits.
Table A1. Fit results of ISRF parameterized as $G$ or $S$ for sample TROPOMI pre-launch calibration measurements at detector edge.

\begin{tabular}{llrrrrr}
\hline Detector & $\begin{array}{l}\text { ISRF } \\
\text { model }\end{array}$ & $\begin{array}{r}w \\
(\mathrm{~nm})\end{array}$ & $k$ & $a_{w}$ & $a_{k}$ & $\begin{array}{r}\text { RMS } \\
(\% o)^{*}\end{array}$ \\
\hline UV & $S_{a_{w}}$ & 0.252 & 8.59 & 0.02 & $\equiv 0$ & 17.9 \\
& $S_{\text {asym }}$ & 0.257 & 9.00 & -0.03 & -1.94 & 17.2 \\
\hline \multirow{2}{*}{ UVIS } & $S_{a_{w}}$ & 0.296 & 2.26 & 0.034 & $\equiv 0$ & 7.0 \\
& $S_{\text {asym }}$ & 0.296 & 2.29 & 0.018 & -0.14 & 6.9 \\
\hline \multirow{2}{*}{ NIR } & $S_{a_{w}}$ & 0.198 & 2.75 & 0.013 & $\equiv 0$ & 5.68 \\
& $S_{\text {asym }}$ & 0.198 & 2.74 & 0.034 & 0.40 & 3.97 \\
\hline \multirow{2}{*}{ SWIR } & $S_{a_{w}}$ & 0.132 & 2.68 & 0.000 & $\equiv 0$ & 13.73 \\
& $S_{\text {asym }}$ & 0.132 & 2.69 & -0.039 & -1.00 & 7.82 \\
\hline
\end{tabular}

* Relative to maximum intensity.

Within this study, we thus focus on $S_{a_{w}}$ for wavelength calibration fits.

\section{Appendix B: Wavelength calibration}

The wavelength calibration of a spectrometer can be performed based on monochromatic stimuli with known wavelength, such as SLSs. However, as the instrument characteristics generally slightly changes during operation, an a posteriori wavelength calibration might be necessary. Within DOAS analysis, wavelength calibration is thus often done based on measured spectra of direct or scattered sunlight, making use of the highly structured Fraunhofer lines. Within this procedure, both the wavelength grid and a parameterized ISRF of the detector can be determined simultaneously.

In the following, we indicate spectral data with high resolution with the tilde symbol. We model a high-resolution spectrum of direct or scattered sunlight by the function $\widetilde{M}(\lambda)$ :

$\widetilde{M}(\lambda)=\widetilde{K}(\lambda) \times \widetilde{A}(\lambda) \times \widetilde{\Pi}_{\mathrm{mul}}(\lambda)+\widetilde{R}(\lambda)+\widetilde{\Pi}_{\mathrm{add}}(\lambda)$.

$\widetilde{K}(\lambda)$ is the solar irradiance. $\widetilde{A}(\lambda)=e^{-\sum c_{j} \times \widetilde{\sigma}_{j}(\lambda)}$ describes absorption by relevant atmospheric gases (like ozone) by Lambert-Beers law. $\widetilde{R}(\lambda)$ accounts for rotational Raman scattering. Any other broadband features such as Mie and Rayleigh scattering, absolute scaling, and offsets are accounted for by the multiplicative and additive polynomials $\widetilde{\Pi}_{\text {mul }}$ and $\widetilde{\Pi}_{\text {add }}$. In case of direct irradiance measurements from satellite, the model function can be simplified as atmospheric absorption and scattering can be omitted. Note that wavelength calibration might as well be performed in terms of optical depths.

The respective spectrum on the instrument's wavelength grid $\lambda_{i}$ and spectral resolution is then given by convolution of $\widetilde{M}(\lambda)$ with the ISRF (here: $S$ ) and interpolation to $\lambda_{i}$, optionally extended by RCS accounting for ISRF changes (see 

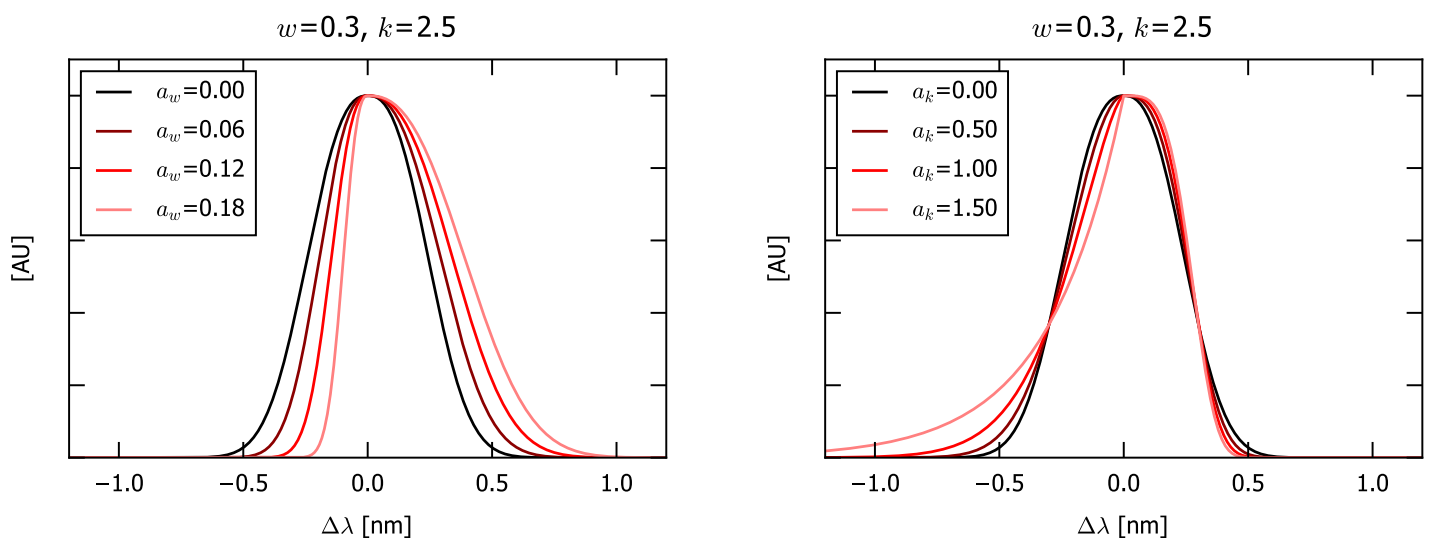

Figure A1. Illustration of the asymmetric super-Gaussian $S_{\text {asym }}$ as defined in Eq. (A1) for $w=0.3 \mathrm{~nm}, k=2.5$, and different values for the asymmetry parameters $a_{w}$ (left) and $a_{k}$ (right). $A_{\text {asym }}$ was set to 1; i.e., the shown ASGs are not yet normalized.
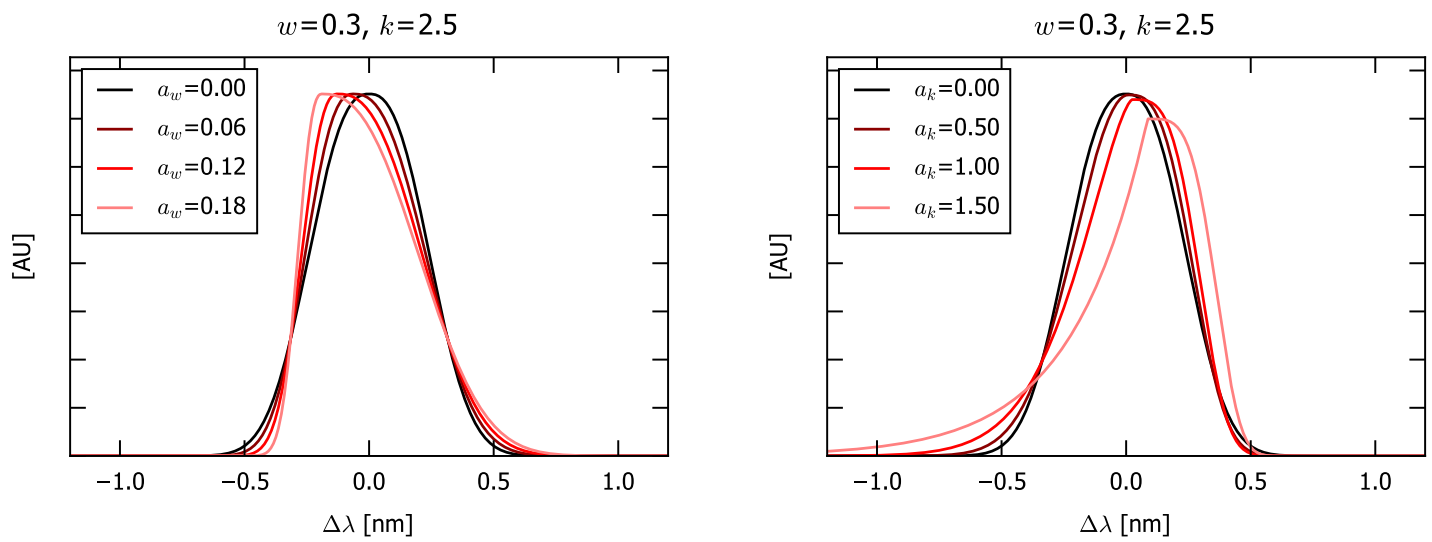

Figure A2. ISRFs based on the $S_{\text {asym }}$ shown in Fig. A1; i.e., $S_{\text {asym }}$ is shifted such that the center of mass of the ISRF is 0 , and normalization is done empirically based on the interval $[-2,2] \mathrm{nm}$.
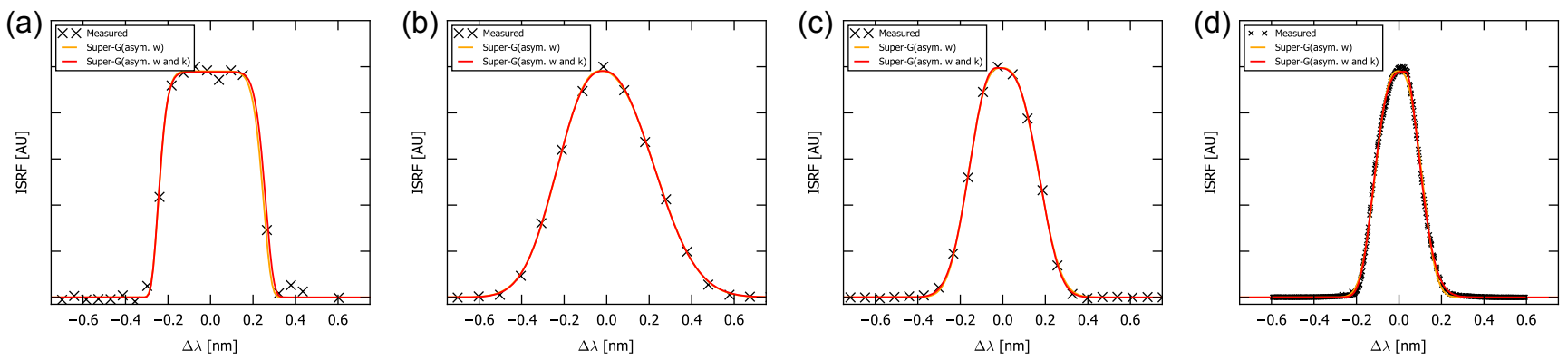

Figure A3. Exemplary TROPOMI ISRF for the UV (a), UVIS (b), NIR (c), and SWIR (d) at the detector edges. Crosses indicate the prelaunch measurements (provided by Antje Ludewig (UV, UVIS, NIR) and Paul Tol (SWIR), personal communication, 2016). Lines show the

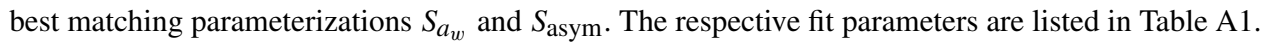


Sect. 2.2):

$M(i)=(S \otimes \tilde{M})\left(\lambda_{i}\right)+\Delta p \times J_{p}$.

For a measured spectrum $I(i)$ with the a priori wavelength grid $\lambda_{i}^{*}$, the difference $M(i)-I(i)$ is minimized ("fitted") by a nonlinear least-squares algorithm (here: using the python LMFit module by Newville et al., 2014), where the calibrated wavelength grid $\lambda_{i}$ is determined from the a priori wavelength grid $\lambda^{*}$ by a linear transformation (allowing for spectral shift and stretch).

Fitted parameters are as follows:

- parameters of $S$ (just $w$ in case of classical Gaussian, $w$ and $k$ in case of SG, and $a_{w}$ in case of asymmetry; see Appendix A);

- column densities $c_{j}$ of the relevant absorbers included in $A$;

- intensity of Raman scattered light $R$;

- polynomial coefficients for $P_{\mathrm{mul}}$ and $P_{\text {add }}$;

- the RCS fit coefficients $\Delta p$;

- shift and stretch of the wavelength grid transformation.

The resulting calibrated wavelength grid and bestmatching ISRF are used to provide the relevant cross sections, necessary for a subsequent DOAS analysis, on the instrument's spectral resolution:

$\sigma_{j}(i)=\left(S \otimes \widetilde{\sigma}_{j}\right)\left(\lambda_{i}\right)$.

Based on the ISRF change $\Delta p$ determined during wavelength calibration, the convolution of cross sections can be corrected accordingly:

$\sigma_{j}(i)=\left(\left(S+\Delta p \partial_{p} S\right) \otimes \widetilde{\sigma}_{j}\right)\left(\lambda_{i}\right)$.

\section{Appendix C: Error of Taylor expansion}

In Sect. 2.2, the effects of ISRF changes are linearized based on a Taylor expansion, where terms of order $\mathcal{O}(2)$ are omitted. We investigate the impact of this approximation for synthetic spectra for typical ISRF settings, i.e., $w_{0}=0.3 \mathrm{~nm}$ and $k=2.3$. For a set of a priori changes of $w$, spectra are derived by convolving the solar atlas with a super-Gaussian ISRF with $w=w_{0}+\Delta w$. Subsequently, a spectral calibration fit is performed for a fixed ISRF with $w=w_{0}$, but including $J_{w}$ in order to account for the change of ISRF width by linearization.
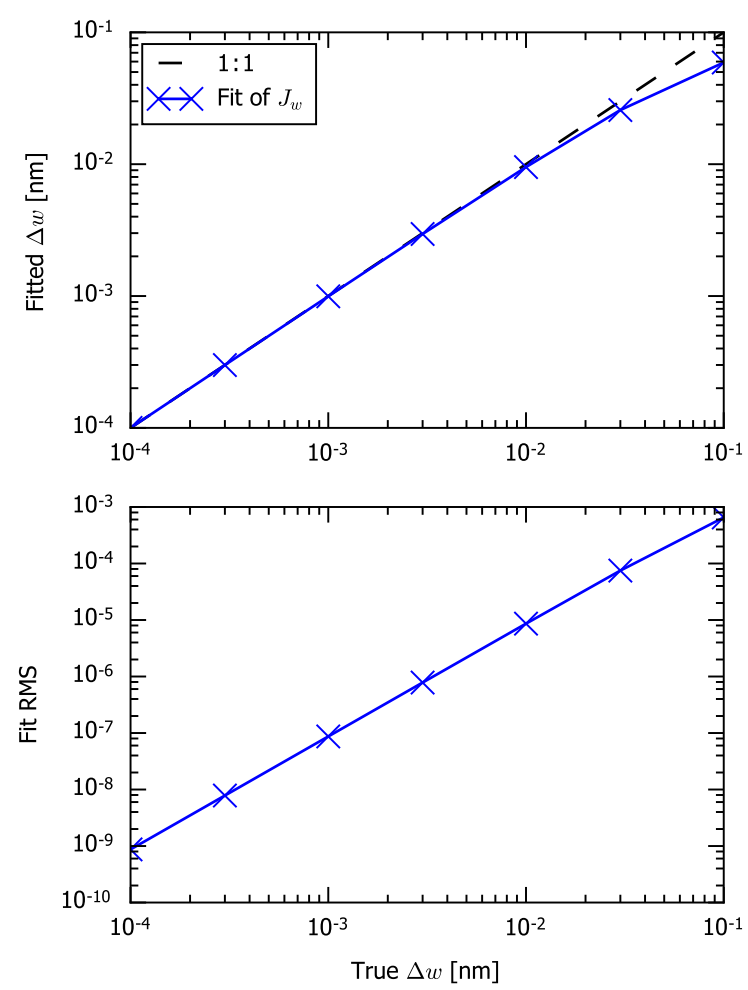

Figure C1. Errors induced by the linearization as investigated for synthetic spectra with width $w=0.3 \mathrm{~nm}+\Delta w$. Top: change of width $\Delta w$, as fitted based on $J_{w}$, versus the true change of $w$. Bottom: fit RMS in dependency of the true change of $w$.

Figure $\mathrm{C} 1$ displays the results of this case study. In the top panel, the fitted $\Delta w$, i.e., the fit coefficient of $J_{w}$, is displayed versus the a priori ("true") change $\Delta w$. In the bottom panel, the respective RMS of the spectral calibration fit is shown.

For small changes of the ISRF width, the linearization works well. For $w=0.303 \mathrm{~nm}$, i.e., a change of $0.003 \mathrm{~nm}$ compared to $w_{0}$, the spectral calibration using $J_{w}$ yields a width of $0.30296 \mathrm{~nm}$, with a RMS below $10^{-6}$. For a true change of $0.03 \mathrm{~nm}$, which corresponds to $10 \%$ of $w_{0}$, the fitted change is $0.026 \mathrm{~nm}$, with a RMS below $10^{-4}$, which is still negligible. 


\section{The Supplement related to this article is available online at doi:10.5194/amt-10-581-2017-supplement.}

Competing interests. The authors declare that they have no conflict of interest.

Acknowledgements. We would like to thank Andreas Richter (IUP Bremen, Germany), Michel van Roozendael (BIRA Brussels, Belgium), Alexander Cede (LuftBlick, Austria), Antje Ludewig (KNMI, de Bilt, the Netherlands), Paul Tol (SRON, Utrecht, the Netherlands), and Kang Sun (Smithsonian Center for Astrophysics, Harvard, Cambridge, MA, USA) for helpful discussions on the topic of ISRF and the parameterization of its change.

Steffen Dörner and Jan Zörner from MPIC Mainz are acknowledged for helpful discussions on Python.

We thank Mingxi Yang from Plymouth Marine Laboratory for setting up and operating the EnviMeS/Avantes MAX-DOAS instrument at Penlee Point.

R. L. Kurucz is acknowledged for providing the solar atlas. GOME-2 spectral measurements are provided by EUMETSAT, Darmstadt. OMI spectral measurements are provided by NASA. TROPOMI ISRF sample measurements are provided by Antje Ludewig and Joost F. C. Smeets (KNMI, de Bilt, the Netherlands) for UV/UVIS/NIR, and by Paul Tol (SRON, Utrecht, the Netherlands) for SWIR. This research was supported by the FP7 project QA4ECV, grant no. 607405.

The article processing charges for this open-access publication were covered by the Max Planck Society.

Edited by: M. Weber

Reviewed by: two anonymous referees

\section{References}

Azam, F. and Richter, A.: GOME2 on MetOp: Followon analysis of GOME2 in orbit degradation, Final report, EUM/CO/09/4600000696/RM, 2015, available at: http://www.doas-bremen.de/reports/gome2_degradation_ follow_up_final_report.pdf (last access: 7 September 2016), 2015.

Beirle, S., Sihler, H., and Wagner, T.: Linearisation of the effects of spectral shift and stretch in DOAS analysis, Atmos. Meas. Tech., 6, 661-675, doi:10.5194/amt-6-661-2013, 2013.

Callies, J., Corpaccioli, E., Eisinger, M., Hahne, A., and Lefebvre, A.: GOME-2 - Metop's second-generation sensor for operational ozone monitoring, ESA Bull.-Eur. Space, 102, 28-36, 2000.

Cede, A.: Manual for Pandora Software Suite, Version 1.6, 2013, available at: http://avdc.gsfc.nasa.gov/pub/tools/Pandora/install/ (last access: 20 September 2016), 2013.

Cede, A.: Manual for Blick Software Suite 1.2, 2017, available at: http://pandonia.net/media/documents/BlickSoftwareSuite_ Manual_v6.pdf, last access: 14 February 2017.
Danckaert, T., Fayt, C., van Roozendael, M., de Smedt, I., Letocart, V., Merlaud, A., and Pinardi, G.: QDOAS Software user manual, available at: http://uv-vis.aeronomie.be/software/QDOAS/ QDOAS_manual.pdf (last access: 9 September 2016), 2015.

Decker, F.: Beam distributions beyond RMS, in: Beam Instrumentation Workshop, edited by: Mackenzie, G. H., Rawnsley, B., and Thomson, J., 550-556, Aip Press, Woodbury, USA, 1995.

de Graaf, M., Sihler, H., Tilstra, L. G., and Stammes, P.: How big is an OMI pixel?, Atmos. Meas. Tech., 9, 3607-3618, doi:10.5194/amt-9-3607-2016, 2016

De Smedt, I., Van Roozendael, M., Stavrakou, T., Müller, J.-F., Lerot, C., Theys, N., Valks, P., Hao, N., and van der A, R.: Improved retrieval of global tropospheric formaldehyde columns from GOME-2/MetOp-A addressing noise reduction and instrumental degradation issues, Atmos. Meas. Tech., 5, 2933-2949, doi:10.5194/amt-5-2933-2012, 2012.

Dirksen, R., Dobber, M., Voors, R., and Levelt, P.: Prelaunch characterization of the Ozone Monitoring Instrument transfer function in the spectral domain, Appl. Optics, 45, 3972-3981, doi:10.1364/ao.45.003972, 2006.

Fleck, J., Morris, J., and Feit, M.: Time-Dependent Propagation of High-Energy Laser-Beams Through Atmosphere, Appl. Phys., 14, 99-115, doi:10.1007/BF00882638, 1977.

Kurucz, R. L., Furenlid, I., Brault, J., and Testerman, L., Solar flux atlas from 296 to $1300 \mathrm{~nm}$, in: National Solar Observatory Atlas, Sunspot, National Solar Observatory, Sunspot, New Mexico, USA, 1984.

Lampel, J., Frieß, U., and Platt, U.: The impact of vibrational Raman scattering of air on DOAS measurements of atmospheric trace gases, Atmos. Meas. Tech., 8, 3767-3787, doi:10.5194/amt-8-3767-2015, 2015.

Lehmann, T.: DOASIS Tutorial, available at: https: //doasis.iup.uni-heidelberg.de/bugtracker/projects/doasis/ files/doasis_tutorial.pdf (last access: 9 September 2016), 2008.

Levelt, P., Van den Oord, G., Dobber, M., Malkki, A., Visser, H., de Vries, J., Stammes, P., Lundell, J., and Saari, H.: The Ozone Monitoring Instrument, IEEE T. Geosci. Remote, 44, 1093 1101, doi:10.1109/TGRS.2006.872333, 2006.

Liu, C., Liu, X., Kowalewski, M. G., Janz, S. J., González Abad, G., Pickering, K. E., Chance, K., and Lamsal, L. N.: Characterization and verification of ACAM slit functions for trace-gas retrievals during the 2011 DISCOVER-AQ flight campaign, Atmos. Meas. Tech., 8, 751-759, doi:10.5194/amt-8-751-2015, 2015.

Miles, G. M., Siddans, R., Kerridge, B. J., Latter, B. G., and Richards, N. A. D.: Tropospheric ozone and ozone profiles retrieved from GOME-2 and their validation, Atmos. Meas. Tech., 8, 385-398, doi:10.5194/amt-8-385-2015, 2015.

Munro, R., Lang, R., Klaes, D., Poli, G., Retscher, C., Lindstrot, R., Huckle, R., Lacan, A., Grzegorski, M., Holdak, A., Kokhanovsky, A., Livschitz, J., and Eisinger, M.: The GOME2 instrument on the Metop series of satellites: instrument design, calibration, and level 1 data processing - an overview, Atmos. Meas. Tech., 9, 1279-1301, doi:10.5194/amt-9-1279-2016, 2016.

Nadarajah, S.: A generalized normal distribution, J. Appl. Stat., 32, 685-694, doi:10.1080/02664760500079464, 2005.

Newville, M., Ingargiola, A., Stensitzki, T., and Allen, D. B.: LMFIT: Non-Linear Least-Square Minimization and Curve-Fitting for Python, doi:10.5281/zenodo.11813, 2014. 
Ozone Monitoring Instrument (OMI): Data User's Guide, available at: http://disc.sci.gsfc.nasa.gov/Aura/additional/documentation/ README.OMI_DUG.pdf (last access: 20 December 2016), 2012.

Platt, U. and Stutz, J.: Differential Optical Absorption Spectroscopy, Springer-Verlag Berlin Heidelberg, Germany, 2008.

Siddans, R., Kerridge, B. J., Latter, B.G., Smeets, J., and Otter, G.: Analysis of GOME-2 Slit function Measurements, Algorithm Theoretical Basis Document, available at: ftp://ftp.eumetsat.int/pub/EPS/out/GOME/ Calibration-Data-Sets/Slit-Function-Key-Data/FM3-Metop-A/ g2_slit_function_analysis_atbd_v2p0.pdf (last access: 14 February 2017), 2006.

Sihler, H., Lübcke, P., Lang, R., Beirle, S., de Graaf, M., Hörmann, C., Lampel, J., Penning de Vries, M., Remmers, J., Trollope, E., Wang, Y., and Wagner, T.: In-operation Field of view Retrieval (IFR) for satellite and ground-based DOAS-type instruments applying coincident high-resolution imager data, Atmos. Meas. Tech. Discuss., doi:10.5194/amt-2016-218, in review, 2016.

Sun, K., Liu, X., Nowlan, C. R., Cai, Z., Chance, K., Frankenberg, C., Lee, R. A. M., Pollock, R., Rosenberg, R., and Crisp, D.: Characterization of the OCO-2 instrument line shape functions using on-orbit solar measurements, Atmos. Meas. Tech. Discuss., doi:10.5194/amt-2016-335, in review, 2016.
Van Roozendael, M., De Smedt, I., Lerot, C., and Theys, N.: Trace gas products at BIRA-IASB Presentation given at the 50th GOME Science Advisory Group Meeting, EUMETSAT, 2627 November 2014, Darmstadt, Germany, 2014.

Veefkind, J. P., Aben, I., McMullan, K., Förster, H., de Vries, J., Otter, G., Claas, J., Eskes, H. J., de Haan, J. F., Kleipool, Q., van Weele, M., Hasekamp, O., Hoogeveen, R., Landgraf, J., Snel, R., Tol, P., Ingmann, P., Voors, R., Kruizinga, B., Vink, R., Visser, H., and Levelt, P. F.: TROPOMI on the ESA Sentinel-5 Precursor: A GMES mission for global observations of the atmospheric composition for climate, air quality and ozone layer applications, Remote Sens. Environ., 120, 70-83, doi:10.1016/j.rse.2011.09.027, 2012.

Yang, M., Bell, T. G., Hopkins, F. E., Kitidis, V., Cazenave, P. W., Nightingale, P. D., Yelland, M. J., Pascal, R. W., Prytherch, J., Brooks, I. M., and Smyth, T. J.: Air-sea fluxes of $\mathrm{CO}_{2}$ and $\mathrm{CH}_{4}$ from the Penlee Point Atmospheric Observatory on the southwest coast of the UK, Atmos. Chem. Phys., 16, 5745-5761, doi:10.5194/acp-16-5745-2016, 2016. 